\title{
Bounded Traveling Wave Solutions and Their Relations for the Generalized HD Type Equation
}

\author{
Qing Meng ${ }^{1}$ and Bin $\mathrm{He}^{2}$ \\ ${ }^{1}$ Department of Physics, Honghe University, Mengzi, Yunnan 661100, China \\ ${ }^{2}$ College of Mathematics, Honghe University, Mengzi, Yunnan 661100, China \\ Correspondence should be addressed to Bin He; hebinmtc@163.com
}

Received 17 March 2016; Revised 18 June 2016; Accepted 4 July 2016

Academic Editor: Andrew Pickering

Copyright (c) 2016 Q. Meng and B. He. This is an open access article distributed under the Creative Commons Attribution License, which permits unrestricted use, distribution, and reproduction in any medium, provided the original work is properly cited.

\begin{abstract}
The generalized HD type equation is studied by using the bifurcation method of dynamical systems. From a dynamic point of view, the existence of different kinds of traveling waves which include periodic loop soliton, periodic cusp wave, smooth periodic wave, loop soliton, cuspon, smooth solitary wave, and kink-like wave is proved and the sufficient conditions to guarantee the existence of the above solutions in different regions of the parametric space are given. Also, all possible exact parametric representations of the bounded waves are presented and their relations are stated.
\end{abstract}

\section{Introduction}

The search for the exact solutions of nonlinear partial differential equations has been one of the most important concerns of mathematicians throughout the world for a long time. Because for understanding the nonlinear phenomena, which are usually described by partial differential equations, the study of the exact solutions is essential. Many powerful methods have been presented for finding the traveling wave solutions of nonlinear partial differential equations, such as the Bäcklund transformation [1], Darboux transformation [2], inverse scattering method [3], Hirota bilinear method [4], Lie group analysis method [5], transformed rational function method [6], extended transformed rational function method [7], exp-function method [8], multiple exp-function algorithm [9], and symbolic computation method [10].

The Harry Dym (HD) equation [11]

$$
u_{t}=2\left(u^{-1 / 2}\right)_{x x x}
$$

is one of the most interesting and exotic nonlinear integrable equations, which was first discovered in an unpublished paper by Harry Dym in 1973. Many fields related to the HD equation are studied. Leo et al. [12] considered the LieBäcklund symmetries for the HD equation. Fuchssteiner et al.
[13] show that the HD equation is completely integrable. Direct links were found between either the $\mathrm{KdV}$ and the HD equations $[14,15]$ or the $\mathrm{mKdV}$ and the HD equations [16]. Different kinds of solutions to the HD equation have been derived by some authors [17-19]. Moreover, the $(2+1)$ dimensional HD equation [20,21], coupled HD hierarchy $[22,23]$, and generalized HD equation $[24,25]$ were studied.

In 2015, Geng et al. [26] first propose a hierarchy of a generalized HD type (gHD type) equation:

$$
u_{t}=2\left(u^{-1 / 2}\right)_{x x x}+2 \tau\left(u^{3 / 2}\right)_{x}
$$

where $\tau \in \mathbb{R}$ is a parameter. Obviously, (2) is reduced to the HD equation (1) with $\tau=0$. Furthermore, under the transformation $u=r^{-2}$, (2) can be written as

$$
r r_{t}=-r^{4} r_{x x x}+3 \tau r_{x}
$$

Geng et al. [26] also stated that (2) and (3) are almost equivalent except for that an unbounded solution might be bounded for the other, and for $\tau=1,-1$, they obtained some exact solutions. To review conveniently, we list them as follows. 
Implicit soliton for $\tau=1$ :

$$
\begin{aligned}
& r_{1}=2-\tanh ^{2}(s), \\
& \xi_{1}=2 \sqrt{2}(s+\tanh (s)),
\end{aligned}
$$
$s \in \mathbb{R}$.

Peakon for $\tau=1$ :

$$
\begin{aligned}
& r_{2}=2-\tanh ^{2}(s+\operatorname{sgn}(s)), \\
& \xi_{2}=2 \sqrt{2}[s+\tanh (s+\operatorname{sgn}(s))-\tanh (\operatorname{sgn}(s))] .
\end{aligned}
$$

(Anti-)kink soliton for $\tau=1$ :

$$
\begin{aligned}
& r_{3}=1+2 \operatorname{csch}^{2}(s), \\
& \xi_{3}=\sqrt{2}(s-2 \operatorname{coth}(s)),
\end{aligned}
$$

$$
s \in(-\infty, 0) \text {, or } s \in(0,+\infty) .
$$

Cuspon for $\tau=1$ :

$$
\begin{aligned}
r_{4}= & -1+2 \tanh ^{2}(s+\operatorname{arcsinh}(\operatorname{sgn}(s))), \\
\xi_{4}= & \sqrt{2} s+2 \operatorname{sgn}(s) \\
& -2 \sqrt{2} \tanh (s+\operatorname{arcsinh}(\operatorname{sgn}(s))),
\end{aligned}
$$

$s \in \mathbb{R}$.

Periodic solution for $\tau=1$ :

$$
\begin{aligned}
& r_{5}=2 \sin ^{2}(s) \\
& \xi_{5}=2 \sqrt{6}[E(s,-1)+F(s,-1)],
\end{aligned}
$$

$$
s \in \mathbb{R},
$$

where $E(\cdot, k)$ and $F(\cdot, k)$ are the elliptic integrals of the second and first kinds, respectively, with the modulus $k$. To be specific,

$$
\begin{aligned}
& E(\varphi, k)=\int_{0}^{\varphi}\left(1-k \sin ^{2} \theta\right)^{1 / 2} d \theta, \\
& F(\varphi, k)=\int_{0}^{\varphi}\left(1-k \sin ^{2} \theta\right)^{-1 / 2} d \theta .
\end{aligned}
$$

Periodic cuspon for $\tau=1$ :

$$
r_{6}=\sqrt{1-\xi^{2}}, \quad \xi \in[-1,1]
$$

Explicit soliton for $\tau=-1$ :

$$
r_{7}=\frac{1}{2}+2 \sinh ^{2}\left(\frac{1}{3} \operatorname{arcsinh}(3 \xi)\right) .
$$

Rational solution for $\tau=-1$ :

$$
r_{8}=\sqrt{1+\xi^{2}}
$$

Periodic solution for $\tau=-1$ :

$$
\begin{aligned}
& r_{9}=\frac{1}{2}(3-\sin (s)), \\
& \xi_{9}=\frac{1}{\sqrt{2}}(3 s+\cos (s)),
\end{aligned}
$$

$$
s \in(-\infty,+\infty) \text {. }
$$

In this paper, by employing the bifurcation method of dynamical systems [27-34], we will obtain some new bounded traveling wave solutions for (3). These solutions contain periodic loop soliton solutions, periodic cusp wave solutions, smooth periodic wave solutions, loop soliton solutions, cuspon solutions, smooth solitary wave solutions, and kink-like wave solutions. Under some parameter conditions, we also show the convergence of certain solutions. For example, when the parameter value varies, the periodic loop soliton solutions converge to the loop soliton solutions.

\section{Preliminaries}

Using a transformation

$$
r(x, t)=r(\xi)=r(x-\omega t),
$$

where $\omega(\neq 0)$ is a constant parameter, (3) can be rewritten as

$$
-\omega r r^{\prime}=-r^{4} r^{\prime \prime \prime}+3 \tau r^{\prime}
$$

where " $/$ " is the derivative with respect to $\xi$. Multiplying both sides of (15) by $-2 r^{-4}$ and integrating with respect to $\xi$ and setting the integral constant as $\omega+2 \tau$, we obtain

$$
2 r^{\prime \prime}=\omega+2 \tau-\omega r^{-2}-2 \tau r^{-3} .
$$

Letting $r(\xi)=1+\phi(\xi)$, we can obtain from (16) that

$$
\phi^{\prime \prime}=\frac{\left[(\omega+2 \tau) \phi^{2}+3(\omega+2 \tau) \phi+2(\omega+3 \tau)\right] \phi}{2(1+\phi)^{3}} .
$$

From (17), we get the following planar dynamical system:

$$
\begin{aligned}
& \frac{d \phi}{d \xi}=y, \\
& \frac{d y}{d \xi}=\frac{\left[(\omega+2 \tau) \phi^{2}+3(\omega+2 \tau) \phi+2(\omega+3 \tau)\right] \phi}{2(1+\phi)^{3}} .
\end{aligned}
$$

Using $d \xi=2(1+\phi)^{3} d \eta$, it carries (18) into the Hamiltonian system

$$
\begin{aligned}
& \frac{d \phi}{d \eta}=2(1+\phi)^{3} y, \\
& \frac{d y}{d \eta}=\left[(\omega+2 \tau) \phi^{2}+3(\omega+2 \tau) \phi+2(\omega+3 \tau)\right] \phi
\end{aligned}
$$

with the following first integral:

$$
H(\phi, y)=y^{2}-(\omega+2 \tau) \phi-\frac{\tau}{(1+\phi)^{2}}-\frac{\omega}{1+\phi}=h
$$


For a fixed $h$, the level curve $H(\phi, y)=h$ defined by (20) determines a set of invariant curves of system (19) which contains different branches of curves. As $h$ is varied, it defines different families of orbits of system (19) with different dynamical behaviors.

Write that $\Delta=\omega^{2}-4 \omega \tau-12 \tau^{2}$. Clearly, when $\tau \neq-(1 / 2) \omega$ and $\Delta>0$, system (19) has three equilibrium points at $(0,0)$, $\left(\phi_{1}, 0\right)$, and $\left(\phi_{2}, 0\right)$ in $\phi$-axis, where $\phi_{1,2}=(-3(\omega+2 \tau) \pm$ $\sqrt{\Delta}) / 2(\omega+2 \tau)$. When $\tau \neq-(1 / 2) \omega$ and $\Delta=0$, system (19) has two equilibrium points at $(0,0)$ and $(-3 / 2,0)$ in $\phi$-axis. When $\Delta<0$ or $\tau=-(1 / 2) \omega$, system (19) has only one equilibrium point at $(0,0)$ in $\phi$-axis. There is not any equilibrium point of system (19) in line $\phi=-1$.

Let $M\left(\phi_{e}, 0\right)$ be the coefficient matrix of the linearized system of (19) at equilibrium point $\left(\phi_{e}, 0\right)$, and then we have $J(0,0)=-4(\omega+3 \tau), J\left(\phi_{1}, 0\right)=(\omega+2 \tau-\sqrt{\Delta})^{3}(\omega-6 \tau-$ $3 \sqrt{\Delta}) / 8(\omega+2 \tau)^{3}, J\left(\phi_{2}, 0\right)=(\omega+2 \tau+\sqrt{\Delta})^{3}(\omega-6 \tau+3 \sqrt{\Delta}) / 8(\omega+$ $2 \tau)^{3}$, and $J(-3 / 2,0)=0$.

For an equilibrium point $\left(\phi_{e}, 0\right)$ of system (19), we know that $\left(\phi_{e}, 0\right)$ is a saddle point if $J\left(\phi_{e}, 0\right)<0$, a center point if $J\left(\phi_{e}, 0\right)>0$, and a cusp if $J\left(\phi_{e}, 0\right)=0$ and the Poincaré index of $\left(\phi_{e}, 0\right)$ is zero.

Notice that for $\Delta=0$, we have $\tau=-(1 / 2) \omega$ and $\tau=$ $(1 / 6) \omega$, respectively. And $J\left(\phi_{1}, 0\right)=0$ implies that $\tau=0$, $\tau=-(1 / 3) \omega$, and $\tau=(1 / 6) \omega$. Therefore, we can obtain four bifurcation curves of (19) as follows:

$$
\begin{aligned}
& C_{1}: \tau=0, \\
& C_{2}: \tau=-\frac{1}{2} \omega, \\
& C_{3}: \tau=-\frac{1}{3} \omega, \\
& C_{4}: \tau=\frac{1}{6} \omega,
\end{aligned}
$$

which divide the $(\omega, \tau)$-parameter plane into 18 subregions.

Since both (18) and (19) have the same first integral (20), then two systems above have the same topological phase portraits. Therefore we can obtain the phase portraits of (18) from that of (19). We show that bifurcation sets and phase portraits of (18) are drawn in Figure 1.

The remainder of this paper is organized as follows. In Section 3, we state our main results for (3). In Section 4, we give the derivations for our main results. Some remarks and a short conclusion will be given in Section 5 .

\section{Main Results}

Let

$$
\begin{aligned}
& h_{0}=H(0,0)=-(\omega+\tau), \\
& h_{1}=H\left(\phi_{1}, 0\right) \\
& =\frac{2\left[3 \omega^{3}+4 \tau \omega^{2}-20 \tau^{2} \omega-32 \tau^{3}-\left(3 \omega^{2}+8 \tau \omega+4 \tau^{2}\right) \sqrt{\Delta}\right]}{(\omega+2 \tau-\sqrt{\Delta})^{2}},
\end{aligned}
$$

$$
\begin{aligned}
& h_{2}=H\left(\phi_{2}, 0\right) \\
& =\frac{2\left[3 \omega^{3}+4 \tau \omega^{2}-20 \tau^{2} \omega-32 \tau^{3}+\left(3 \omega^{2}+8 \tau \omega+4 \tau^{2}\right) \sqrt{\Delta}\right]}{(\omega+2 \tau+\sqrt{\Delta})^{2}},
\end{aligned}
$$

and then our main results are stated in the following seven propositions.

Proposition 1. When $\omega<0,0<\tau<-(1 / 2) \omega, h \in$ $\left(-\infty, h_{1}\right),(3)$ has a periodic loop soliton solution as follows:

$$
\begin{aligned}
r(\xi) & =\left(1+\gamma_{1}\right)+\left(\gamma_{2}-\gamma_{1}\right) \operatorname{sn}^{2}(\bar{\omega} \chi, k), \\
\xi & =\left(1+\gamma_{3}\right) \chi+\frac{\gamma_{1}-\gamma_{3}}{\bar{\omega}} E(\operatorname{am}(\bar{\omega} \chi, k), k),
\end{aligned}
$$

where $\bar{\omega}=(1 / 2) \sqrt{(\omega+2 \tau)\left(\gamma_{3}-\gamma_{1}\right)}, k=$ $\sqrt{\left(\gamma_{1}-\gamma_{2}\right) /\left(\gamma_{1}-\gamma_{3}\right)}, \operatorname{sn}(\cdot, k)$ is the Jacobian elliptic function with the modulus $k, \operatorname{am}\left(u_{1}, k\right)$ reads amplitude $u_{1}$ [35], $\gamma_{1}, \gamma_{2}, \gamma_{3}\left(\gamma_{3}<\gamma_{2}<\gamma_{1}\right)$ are three real roots of $(\omega+2 \tau) X^{3}+(2 \omega+4 \tau+h) X^{2}+2(\omega+\tau+h) X+(\omega+\tau+h)=0$, and $\chi$ is a new parametric variable.

When $\omega<0, \tau=-(1 / 2) \omega, h \in(-\infty, 0)$, (3) has a periodic loop soliton solution as follows:

$$
\begin{aligned}
r(\xi) & =1+\gamma_{1} \cos ^{2}(\bar{\omega} \chi)+\gamma_{2} \sin ^{2}(\bar{\omega} \chi), \\
\xi & =\left(1+\frac{\gamma_{1}+\gamma_{2}}{2}\right) \chi+\frac{\gamma_{1}-\gamma_{2}}{4 \bar{\omega}} \sin (2 \bar{\omega} \chi),
\end{aligned}
$$

where $\bar{\omega}=(1 / 2) \sqrt{-h}, \gamma_{1,2}=-(\omega+2 h \pm \sqrt{\omega(\omega+2 h)}) / 2 h$.

When $\omega<0, \tau>-(1 / 2) \omega($ or $\omega>0, \tau>0), h \in$ $\left(-\infty, h_{0}\right),(3)$ has a periodic loop soliton solution as follows:

$$
\begin{aligned}
r(\xi) & =\left(1+\gamma_{3}\right)+\left(\gamma_{2}-\gamma_{3}\right) \operatorname{sn}^{2}(\bar{\omega} \chi, k), \\
\xi & =\left(1+\gamma_{1}\right) \chi+\frac{\gamma_{3}-\gamma_{1}}{\bar{\omega}} E(\operatorname{am}(\bar{\omega} \chi, k), k),
\end{aligned}
$$

where $\bar{\omega}=(1 / 2) \sqrt{(\omega+2 \tau)\left(\gamma_{1}-\gamma_{3}\right)}, k=$ $\sqrt{\left(\gamma_{2}-\gamma_{3}\right) /\left(\gamma_{1}-\gamma_{3}\right)}, \gamma_{1}, \gamma_{2}, \gamma_{3}\left(\gamma_{3}<\gamma_{2}<\gamma_{1}\right)$ are three real roots of $(\omega+2 \tau) X^{3}+(2 \omega+4 \tau+h) X^{2}+2(\omega+\tau+h) X+(\omega+\tau+h)=$ 0 .

Example 2. Taking $\omega=-1.2, \tau=0.6, h=-2.0$, we get the approximations of $\bar{\omega}, \gamma_{1}, \gamma_{2}$ in formula (24), where $\bar{\omega} \doteq$ $0.707107, \gamma_{1} \doteq-0.6755002$, and $\gamma_{2} \doteq-1.9244998$. The profile of (24) is shown in Figure 2.

Proposition 3. When $\omega<0, \tau=0, h \in(-\infty, 3 \omega)$, (3) has a periodic cusp wave solution as follows:

$$
\begin{aligned}
r(\xi) & =\left(1+\gamma_{1}\right) \operatorname{sn}^{2}(\bar{\omega} \chi, k), \\
\xi & =\left(1+\gamma_{2}\right) \chi-\frac{1+\gamma_{2}}{\bar{\omega}} E(\operatorname{am}(\bar{\omega} \chi, k), k),
\end{aligned}
$$

where $\bar{\omega}=(1 / 2) \sqrt{\omega\left(1+\gamma_{2}\right)}, k=\sqrt{\left(1+\gamma_{1}\right) /\left(1+\gamma_{2}\right)}$, and $\gamma_{1,2}=-(h+\omega \pm \sqrt{(h+\omega)(h-3 \omega)}) / 2 \omega$. 


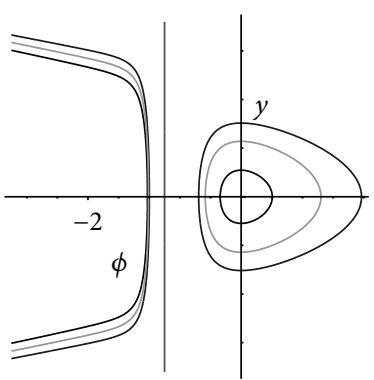

(a) $\omega<0, \tau<(1 / 6) \omega$

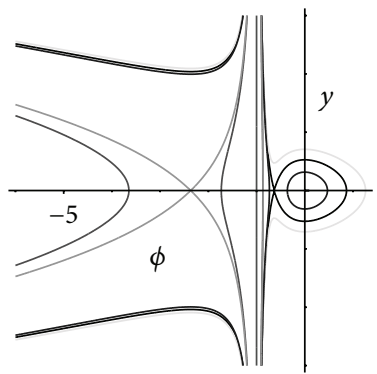

(e) $\omega<0,0<\tau<-(1 / 3) \omega$

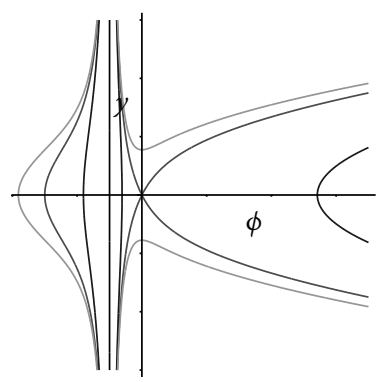

(i) $\omega<0, \tau>-(1 / 2) \omega$

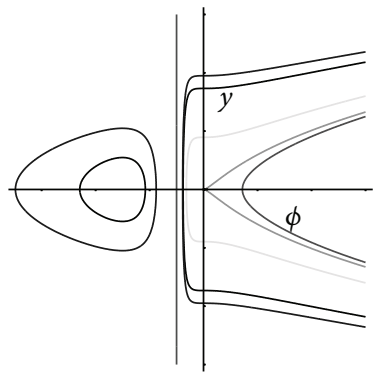

(m) $\omega>0, \tau=-(1 / 3) \omega$

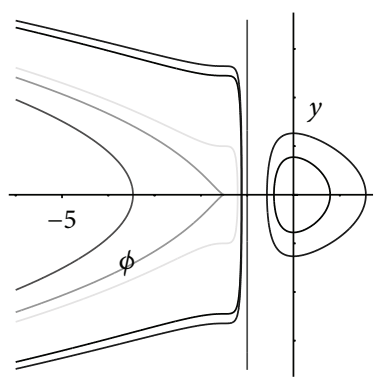

(b) $\omega<0, \tau=(1 / 6) \omega$

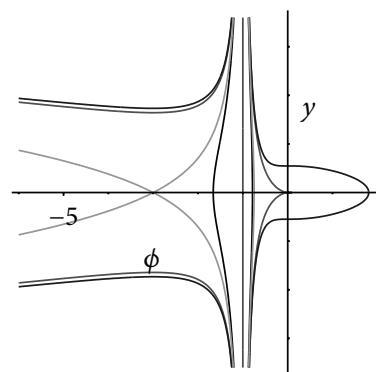

(f) $\omega<0, \tau=-(1 / 3) \omega$

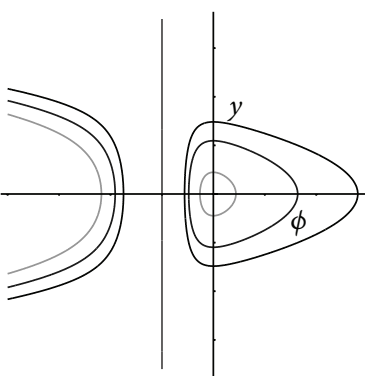

(j) $\omega>0, \tau<-(1 / 2) \omega$

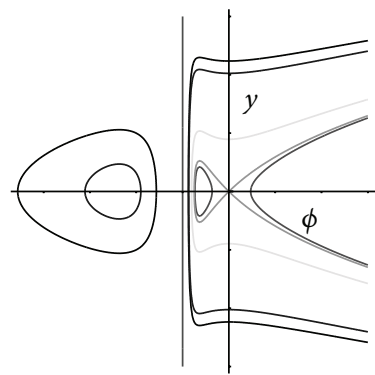

(n) $\omega>0,-(1 / 3) \omega<\tau<0$

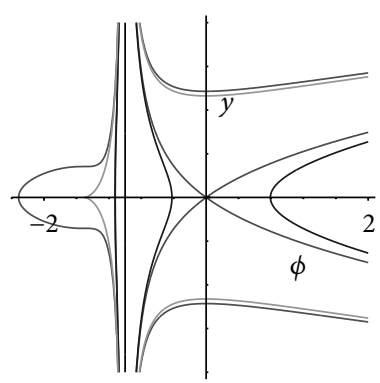

(q) $\omega>0, \tau=(1 / 6) \omega$

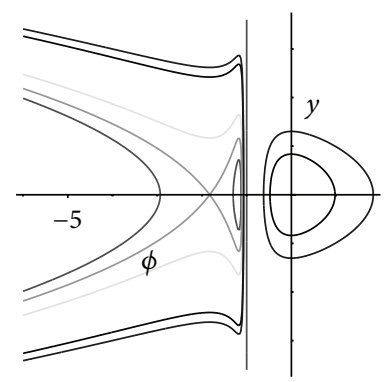

(c) $\omega<0,(1 / 6) \omega<\tau<0$

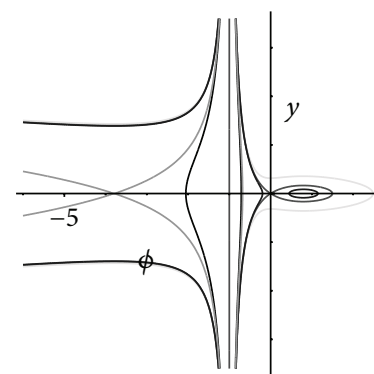

(g) $\omega<0,-(1 / 3) \omega<\tau<$ $-(1 / 2) \omega$

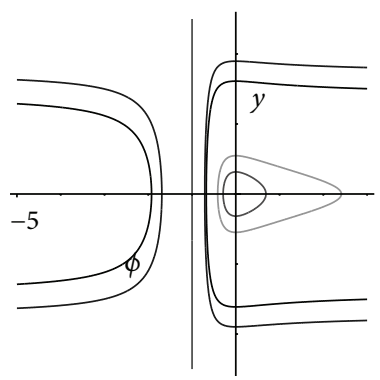

(k) $\omega>0, \tau=-(1 / 2) \omega$

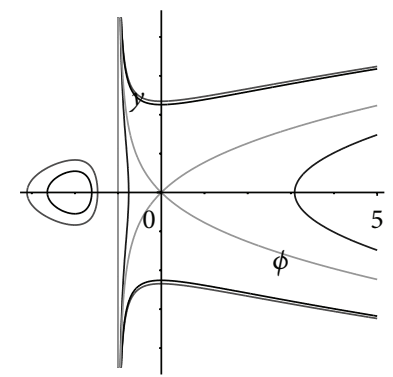

(o) $\omega>0, \tau=0$

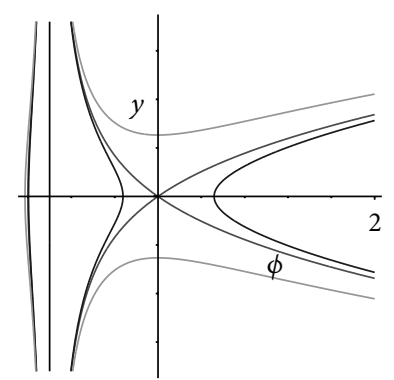

(r) $\omega>0, \tau>(1 / 6) \omega$

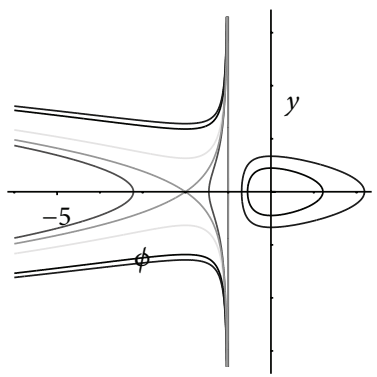

(d) $\omega<0, \tau=0$

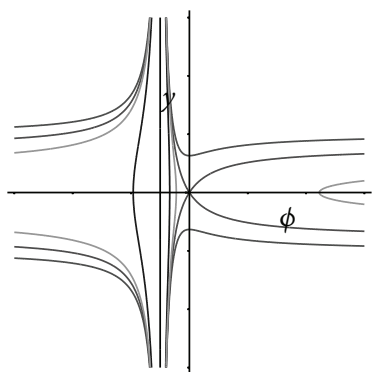

(h) $\omega<0, \tau=-(1 / 2) \omega$

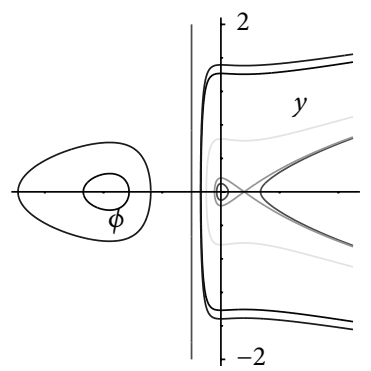

(l) $\omega>0,-(1 / 2) \omega<\tau<$ $-(1 / 3) \omega$

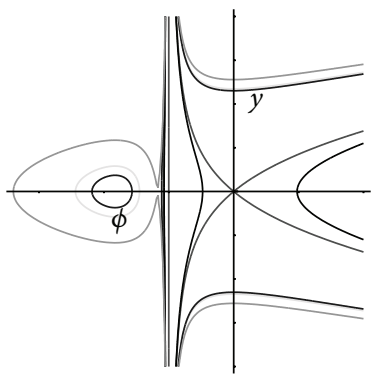

(p) $\omega>0,0<\tau<(1 / 6) \omega$

Figure 1: Bifurcation sets and phase portraits of (18). 


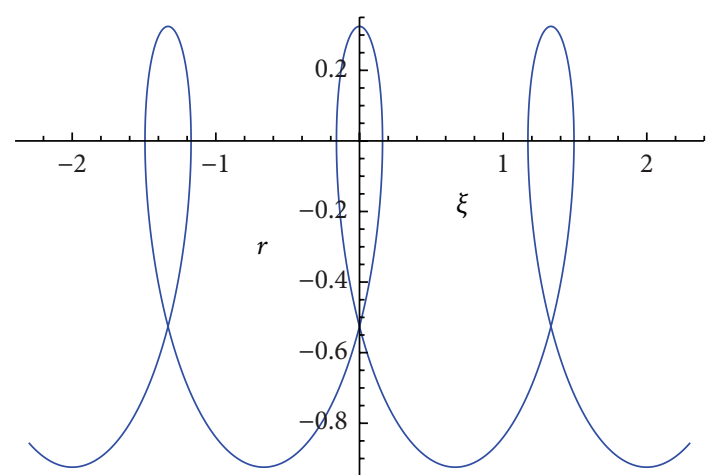

Figure 2: Periodic loop soliton of (3).

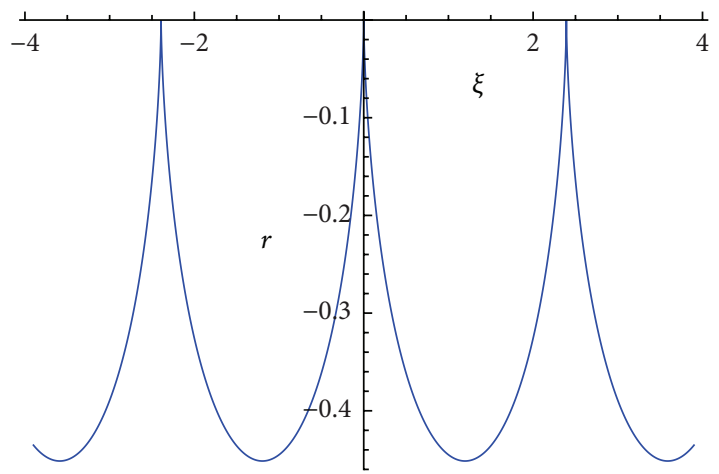

Figure 3: Periodic cusp wave of (3).

When $\omega>0, \tau=0, h \in(-\infty,-\omega)$, (3) has a periodic cusp wave solution as follows:

$$
\begin{aligned}
r(\xi) & =\left(1+\gamma_{2}\right) \operatorname{sn}^{2}(\bar{\omega} \chi, k), \\
\xi & =\left(1+\gamma_{1}\right) \chi-\frac{1+\gamma_{1}}{\bar{\omega}} E(\operatorname{am}(\bar{\omega} \chi, k), k),
\end{aligned}
$$

where $\bar{\omega}=(1 / 2) \sqrt{\omega\left(1+\gamma_{1}\right)}, k=\sqrt{\left(1+\gamma_{2}\right) /\left(1+\gamma_{1}\right)}$, and $\gamma_{1,2}=-\left(h+\omega \mp \sqrt{h^{2}-2 \omega h-3 \omega^{2}}\right) / 2 \omega$.

Example 4. Taking $\omega=-1.2, h=-4.4$, we get the approximations of $\gamma_{1}, \gamma_{2}, \bar{\omega}, k$ in formula (26), where $\gamma_{1} \doteq$ $-1.451416230, \gamma_{2} \doteq-3.215250437, \bar{\omega} \doteq 0.815215$, and $k \doteq$ 0.451416 . The profile of (26) is shown in Figure 3.

Proposition 5. When $\omega<0,(1 / 6) \omega<\tau<0, h \in$ $\left(h_{2}, h_{1}\right)$ (or $\omega<0,0<\tau<-(1 / 3) \omega, h \in\left(h_{0}, h_{2}\right)$ or $\omega<0$, $\left.-(1 / 3) \omega<\tau<-(1 / 2) \omega, h \in\left(h_{2}, h_{0}\right)\right)$, (3) has a smooth periodic wave solution as follows:

$$
\begin{aligned}
r(\xi) & =\left(1+\gamma_{1}\right)+\left(\gamma_{2}-\gamma_{1}\right) \operatorname{sn}^{2}(\bar{\omega} \chi, k), \\
\xi & =\left(1+\gamma_{3}\right) \chi+\frac{\gamma_{1}-\gamma_{3}}{\bar{\omega}} E(\operatorname{am}(\bar{\omega} \chi, k), k),
\end{aligned}
$$

where $\bar{\omega}$ and $k$ are given in (23). $\gamma_{1}, \gamma_{2}, \gamma_{3}\left(\gamma_{3}<\gamma_{2}<\gamma_{1}\right)$ are three real roots of $(\omega+2 \tau) X^{3}+(2 \omega+4 \tau+h) X^{2}+2(\omega+\tau+$ $h) X+(\omega+\tau+h)=0$.

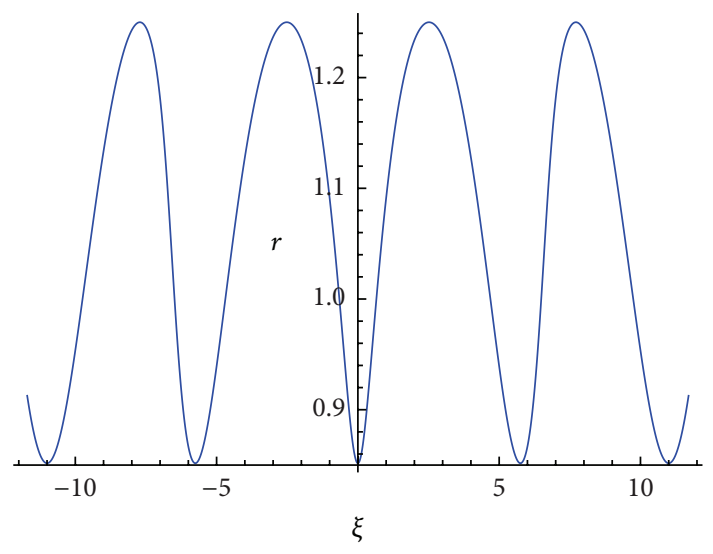

FIgURE 4: Smooth periodic wave of (3).

When $\omega>0,-(1 / 2) \omega<\tau<-(1 / 3) \omega, h \in$ $\left(h_{0}, h_{1}\right)\left(\right.$ or $\omega>0,-(1 / 3) \omega<\tau<0, h \in\left(h_{1}, h_{0}\right)$ or $\omega>$ $\left.0,0<\tau<(1 / 6) \omega, h \in\left(h_{2}, h_{1}\right)\right)$, (3) has a smooth periodic wave solution as follows:

$$
\begin{aligned}
r(\xi) & =\left(1+\gamma_{3}\right)+\left(\gamma_{2}-\gamma_{3}\right) \operatorname{sn}^{2}(\bar{\omega} \chi, k), \\
\xi & =\left(1+\gamma_{1}\right) \chi+\frac{\gamma_{3}-\gamma_{1}}{\bar{\omega}} E(\operatorname{am}(\bar{\omega} \chi, k), k),
\end{aligned}
$$

where $\bar{\omega}=(1 / 2) \sqrt{(\omega+2 \tau)\left(\gamma_{1}-\gamma_{3}\right)}, k=\sqrt{\left(\gamma_{2}-\gamma_{3}\right) /\left(\gamma_{1}-\gamma_{3}\right)}$, $\gamma_{1}, \gamma_{2}, \gamma_{3}\left(\gamma_{3}<\gamma_{2}<\gamma_{1}\right)$ are three real roots of $(\omega+2 \tau) X^{3}+$ $(2 \omega+4 \tau+h) X^{2}+2(\omega+\tau+h) X+(\omega+\tau+h)=0$.

Example 6. Taking $\omega=1.2, \tau=-0.5, h=-0.69$, we get the approximations of $\gamma_{1}, \gamma_{2}, \gamma_{3}, \bar{\omega}, k$ in formula (29), where $\gamma_{1} \doteq 1.34833, \gamma_{2} \doteq 0.25, \gamma_{3} \doteq-0.148331, \bar{\omega} \doteq 1.02355$, and $k \doteq 0.515894$. The profile of (29) is shown in Figure 4 .

Proposition 7. When $\omega<0,0<\tau<-(1 / 2) \omega$, (3) has a loop soliton solution as follows:

$$
\begin{aligned}
r(\xi) & =\left(1+\phi_{a}\right)+\left(\phi_{1}-\phi_{a}\right) \tanh ^{2}(\bar{\omega} \chi), \\
\xi & =\frac{1}{\sqrt{\Omega}}\left(\chi+2 \sqrt{\phi_{a}-\phi_{1}} \tanh (\bar{\omega} \chi)\right),
\end{aligned}
$$

where $\phi_{a}=-(\sqrt{\Delta}-\omega) /(\sqrt{\Delta}-\omega+2 \tau), \Omega=2(\omega+2 \tau)^{2}(\sqrt{\Delta}-$ $(\omega-2 \tau)) /(\sqrt{\Delta}-(\omega+2 \tau))^{2}, \bar{\omega}=\sqrt{\phi_{a}-\phi_{1}} / 2\left(1+\phi_{1}\right)$.

When $\omega<0, \tau>-(1 / 2) \omega$ (or $\omega>0, \tau>0)$, (3) has a loop soliton solution as follows:

$$
\begin{array}{r}
r(\xi)=1+\phi_{b} \operatorname{sech}^{2}(\bar{\omega} \chi), \\
\xi=\chi+\frac{\phi_{b}}{\bar{\omega}} \tanh (\bar{\omega} \chi),
\end{array}
$$

where $\phi_{b}=-(\omega+3 \tau) /(\omega+2 \tau), \bar{\omega}=(1 / 2) \sqrt{-\phi_{b}(\omega+2 \tau)}$.

Example 8. Taking $\omega=1.2, \tau=0.1$, we get the approximations of $\phi_{b}, \bar{\omega}$ in formula (31), where $\phi_{b} \doteq-1.07143$, $\bar{\omega} \doteq 0.612372$. The profile of (31) is shown in Figure 5 . 


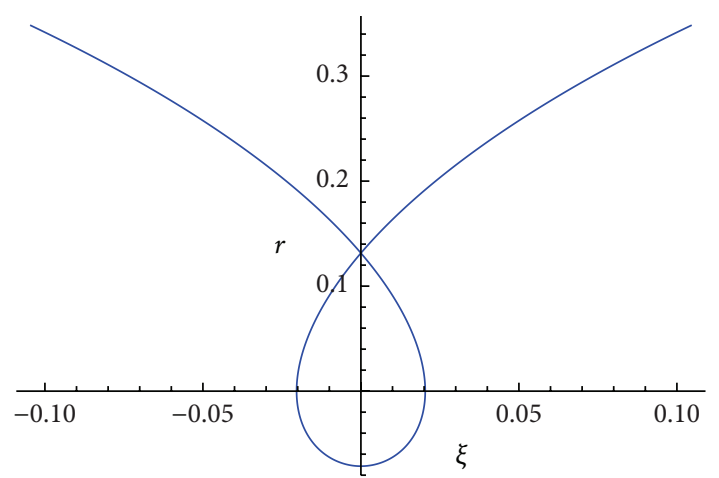

Figure 5: Loop soliton of (3).

Proposition 9. When $\omega<0, \tau=0$, (3) has a cuspon solution as follows:

$$
\begin{aligned}
r(\xi) & =-\tanh ^{2}(\bar{\omega} \chi), \\
\xi & =-\chi+\frac{1}{\bar{\omega}} \tanh (\bar{\omega} \chi),
\end{aligned}
$$

where $\bar{\omega}=(1 / 2) \sqrt{-\omega}$.

When $\omega>0, \tau=0$, (3) has a cuspon solution as follows:

$$
\begin{aligned}
r(\xi) & =\tanh ^{2}(\bar{\omega} \chi), \\
\xi & =\chi-\frac{1}{\bar{\omega}} \tanh (\bar{\omega} \chi),
\end{aligned}
$$

where $\bar{\omega}=(1 / 2) \sqrt{\omega}$.

Example 10. Taking $\omega=1.2$, we get the approximations of $\bar{\omega}$ in formula (33), where $\bar{\omega} \doteq 0.547723$. The profile of (33) is shown in Figure 6.

Proposition 11. When $\omega<0,(1 / 6) \omega<\tau<0$ (or $\omega>0$, $-(1 / 2) \omega<\tau<-(1 / 3) \omega$ or $\omega>0,0<\tau<(1 / 6) \omega)$, (3) has a smooth solitary wave solution as follows:

$$
\begin{aligned}
r(\xi) & =\left(1+\phi_{a}\right)+\left(\phi_{1}-\phi_{a}\right) \tanh ^{2}(\bar{\omega} \chi), \\
\xi & =\left(1+\phi_{1}\right) \chi+\frac{\phi_{a}-\phi_{1}}{\bar{\omega}} \tanh (\bar{\omega} \chi),
\end{aligned}
$$

where $\bar{\omega}=(1 / 2) \sqrt{\Omega\left(\phi_{a}-\phi_{1}\right)}$ and $\phi_{a}$ and $\Omega$ are given in (30).

When $\omega<0,0<\tau<-(1 / 3) \omega$, (3) has a smooth solitary wave solution as follows:

$$
\begin{aligned}
r(\xi) & =\left(1+\phi_{c}\right)+\left(\phi_{2}-\phi_{c}\right) \tanh ^{2}(\bar{\omega} \chi), \\
\xi & =\left(1+\phi_{2}\right) \chi+\frac{\phi_{c}-\phi_{2}}{\bar{\omega}} \tanh (\bar{\omega} \chi),
\end{aligned}
$$

where $\phi_{c}=-(\sqrt{\Delta}+\omega) /(\sqrt{\Delta}+\omega-2 \tau), \Omega=-2(\omega+2 \tau)^{2}(\sqrt{\Delta}+$ $(\omega-2 \tau)) /(\sqrt{\Delta}+(\omega+2 \tau))^{2}, \bar{\omega}=(1 / 2) \sqrt{\Omega\left(\phi_{c}-\phi_{2}\right)}$.

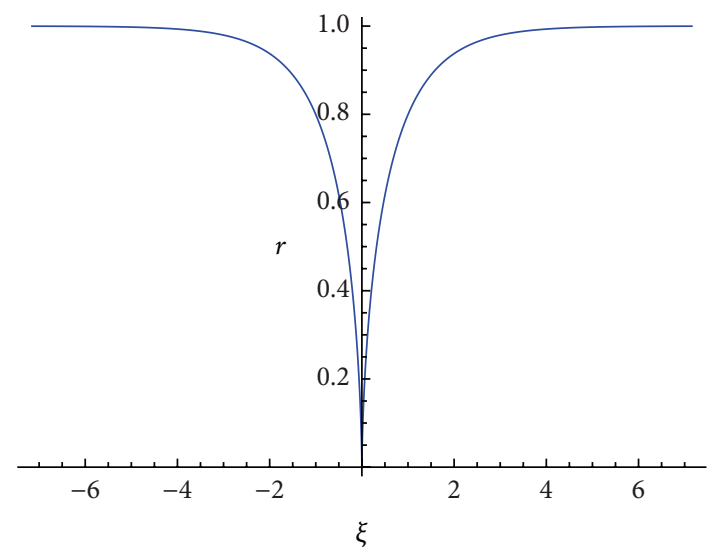

Figure 6: Cuspon of (3).

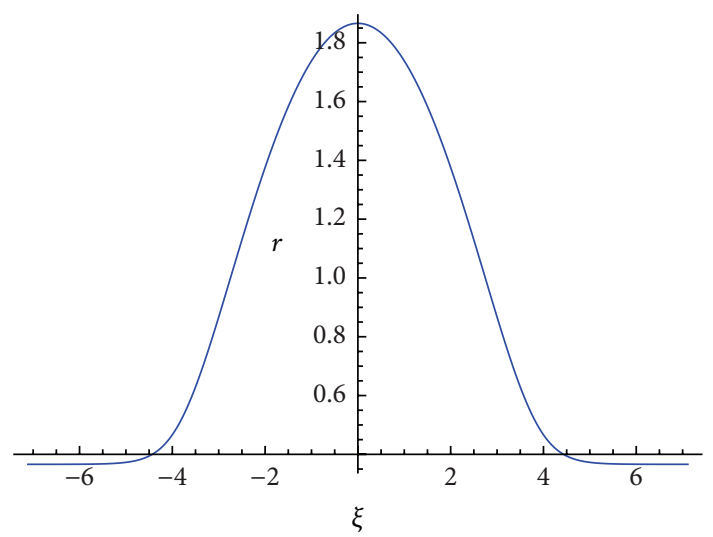

Figure 7: Smooth solitary wave of (3).

When $\omega<0,-(1 / 3) \omega<\tau<-(1 / 2) \omega$ (or $\omega>$ $0,-(1 / 3) \omega<\tau<0)$, (3) has a smooth solitary wave solution as follows:

$$
\begin{aligned}
r(\xi) & =1+\phi_{b} \operatorname{sech}^{2}(\bar{\omega} \chi), \\
\xi & =\chi+\frac{\phi_{b}}{\bar{\omega}} \tanh (\bar{\omega} \chi),
\end{aligned}
$$

where $\phi_{b}$ and $\bar{\omega}$ are given in (31).

Example 12. Taking $\omega=-1.2, \tau=0.2$, we get the approximations of $\phi_{2}, \phi_{c}, \Omega, \bar{\omega}$ in formula (35), where $\phi_{2} \doteq$ $-0.633975, \phi_{c} \doteq 0.866025, \Omega \doteq 0.8, \bar{\omega} \doteq 0.547723$. The profile of (35) is shown in Figure 7.

Proposition 13. When $\omega<0,0<\tau<-(1 / 3) \omega$, (3) has two kink-like wave solutions of implicit expression as follows:

$$
\begin{aligned}
& \xi= \frac{2}{\sqrt{\Omega}}\left(\bar{\Omega}-\sqrt{1+\phi_{c}-r(\xi)}\right. \\
&\left.-\frac{1+\phi_{2}}{\sqrt{\phi_{c}-\phi_{2}}} \tanh ^{-1}\left(\sqrt{\frac{\phi_{c}-\phi_{2}}{1+\phi_{c}-r(\xi)}}\right)\right), \\
&-\infty<\xi<\xi_{1},
\end{aligned}
$$




$$
\begin{aligned}
& \xi= \frac{2}{\sqrt{\Omega}}\left(\bar{\Omega}-\sqrt{1+\phi_{c}-r(\xi)}\right. \\
&\left.-\frac{1+\phi_{2}}{\sqrt{\phi_{c}-\phi_{2}}} \tanh ^{-1}\left(\sqrt{\frac{\phi_{c}-\phi_{2}}{1+\phi_{c}-r(\xi)}}\right)\right), \\
&-\xi_{1}<\xi<+\infty,
\end{aligned}
$$

where $\bar{\Omega}=\sqrt{\phi_{c}-\phi_{0}}+\left(\left(1+\phi_{2}\right) /\right.$ $\left.\sqrt{\phi_{c}-\phi_{2}}\right) \tanh ^{-1}\left(\sqrt{\left(\phi_{c}-\phi_{2}\right) /\left(\phi_{c}-\phi_{0}\right)}\right),-1<\phi_{0}<\phi_{2}, \phi_{c}$ and $\Omega$ are given in (35), and $\xi_{1}=(2 / \sqrt{\Omega})\left(\bar{\Omega}-\sqrt{1+\phi_{c}}-((1+\right.$ $\left.\left.\left.\phi_{2}\right) / \sqrt{\phi_{c}-\phi_{2}}\right) \tanh ^{-1}\left(\sqrt{\left(\phi_{c}-\phi_{2}\right) /\left(1+\phi_{c}\right)}\right)\right)$.

When $\omega<0, \tau=-(1 / 3) \omega$, (3) has two kink-like wave solutions of implicit expression as follows:

$$
\begin{array}{r}
\xi=\frac{1}{\bar{\omega}}\left(\sqrt{-\phi_{0}}+\frac{1}{\sqrt{-\phi_{0}}}-\sqrt{1-r(\xi)}-\frac{1}{\sqrt{1-r(\xi)}}\right), \\
-\infty<\xi<\xi_{2},
\end{array}
$$

$\xi$

$$
\begin{array}{r}
=-\frac{1}{\bar{\omega}}\left(\sqrt{-\phi_{0}}+\frac{1}{\sqrt{-\phi_{0}}}-\sqrt{1-r(\xi)}-\frac{1}{\sqrt{1-r(\xi)}}\right), \\
-\xi_{2}<\xi<+\infty,
\end{array}
$$

where $\bar{\omega}=(1 / 6) \sqrt{-3 \omega}, \xi_{2}=\left(1-\phi_{0}-2 \sqrt{-\phi_{0}}\right) / \bar{\omega} \sqrt{-\phi_{0}},-1<$ $\phi_{0}<0$.

When $\omega<0,-(1 / 3) \omega<\tau<-(1 / 2) \omega$, (3) has two kinklike wave solutions of implicit expression as follows:

$$
\begin{aligned}
\xi= & \frac{2}{\sqrt{-\omega}}\left(\bar{\Omega}-\sqrt{1+\phi_{b}-r(\xi)}\right. \\
& -\frac{1}{\sqrt{\phi_{b}}} \tanh ^{-1}\left(\sqrt{\left.\left.\frac{\phi_{b}}{1+\phi_{b}-r(\xi)}\right)\right),}-\infty<\xi<\xi_{3},\right. \\
\xi= & -\frac{2}{\sqrt{-\omega}}\left(\bar{\Omega}-\sqrt{1+\phi_{b}-r(\xi)}\right. \\
& \left.-\frac{1}{\sqrt{\phi_{b}}} \tanh ^{-1}\left(\sqrt{\frac{\phi_{b}}{1+\phi_{b}-r(\xi)}}\right)\right),
\end{aligned}
$$

$-\xi_{3}<\xi<+\infty$,

where $\bar{\Omega}=\sqrt{\phi_{b}-\phi_{0}}+\left(1 / \sqrt{\phi_{b}}\right) \tanh ^{-1}\left(\sqrt{\phi_{b} /\left(\phi_{b}-\phi_{0}\right)}\right), \xi_{3}=$ $(2 / \sqrt{-\omega})\left(\bar{\Omega}-\sqrt{1+\phi_{b}}-\left(1 / \sqrt{\phi_{b}}\right) \tanh ^{-1}\left(\sqrt{\phi_{b} /\left(1+\phi_{b}\right)}\right)\right),-1<$ $\phi_{0}<0$ and $\phi_{b}$ is given in (31).
When $\omega<0, \tau=-(1 / 2) \omega$, (3) has two kink-like wave solutions of implicit expression as follows:

$$
\begin{aligned}
& \xi=\frac{1}{\bar{\omega}}\left(r(\xi)-1-\phi_{0}-\ln \left(\frac{\phi_{0}}{r(\xi)-1}\right)\right),-\infty<\xi<\xi_{4}, \\
& \xi=-\frac{1}{\bar{\omega}}\left(r(\xi)-1-\phi_{0}-\ln \left(\frac{\phi_{0}}{r(\xi)-1}\right)\right),
\end{aligned}
$$

$$
-\xi_{4}<\xi<+\infty
$$

where $\bar{\omega}=(1 / 2) \sqrt{-2 \omega}, \xi_{4}=-(1 / \bar{\omega})\left(1+\phi_{0}+\ln \left(-\phi_{0}\right)\right),-1<$ $\phi_{0}<0$.

When $\omega>0,0<\tau<(1 / 6) \omega$, (3) has two kink-like wave solutions of implicit expression as follows:

$$
\begin{aligned}
\xi= & \frac{2}{\sqrt{\Omega}}\left(\bar{\Omega}-\sqrt{r(\xi)-1-\phi_{a}}\right. \\
& \left.+\frac{1+\phi_{1}}{\sqrt{\phi_{1}-\phi_{a}}} \tanh ^{-1}\left(\sqrt{\frac{\phi_{1}-\phi_{a}}{r(\xi)-1-\phi_{a}}}\right)\right),
\end{aligned}
$$$$
-\infty<\xi<\xi_{5}
$$

$$
\begin{aligned}
\xi= & -\frac{2}{\sqrt{\Omega}}\left(\bar{\Omega}-\sqrt{r(\xi)-1-\phi_{a}}\right. \\
& \left.+\frac{1+\phi_{1}}{\sqrt{\phi_{1}-\phi_{a}}} \tanh ^{-1}\left(\sqrt{\frac{\phi_{1}-\phi_{a}}{r(\xi)-1-\phi_{a}}}\right)\right),
\end{aligned}
$$

$$
-\xi_{5}<\xi<+\infty
$$

where $\bar{\Omega}=\sqrt{\phi_{0}-\phi_{a}}-\left(\left(1+\phi_{1}\right) /\right.$ $\left.\sqrt{\phi_{1}-\phi_{a}}\right) \tanh ^{-1}\left(\sqrt{\left(\phi_{1}-\phi_{a}\right) /\left(\phi_{0}-\phi_{a}\right)}\right), \phi_{1}<\phi_{0}<-1, \phi_{a}$ and $\Omega$ are given in (30), and $\xi_{5}=(2 / \sqrt{\Omega})\left(\bar{\Omega}-\sqrt{-\left(1+\phi_{a}\right)}+\right.$ $\left.\left(\left(1+\phi_{1}\right) / \sqrt{\phi_{1}-\phi_{a}}\right) \tanh ^{-1}\left(\sqrt{\left(\phi_{a}-\phi_{1}\right) /\left(1+\phi_{a}\right)}\right)\right)$.

When $\omega>0, \tau=(1 / 6) \omega$, (3) has two kink-like wave solutions of implicit expression as follows:

$$
\begin{aligned}
\xi= & \frac{1}{\bar{\omega}}\left(\sqrt{\phi_{0}+\frac{3}{2}}+\frac{1}{2 \sqrt{\phi_{0}+3 / 2}}-\sqrt{r(\xi)+\frac{1}{2}}\right. \\
& \left.-\frac{1}{2 \sqrt{r(\xi)+1 / 2}}\right), \quad-\infty<\xi<\xi, \\
\xi= & -\frac{1}{\bar{\omega}}\left(\sqrt{\phi_{0}+\frac{3}{2}}+\frac{1}{2 \sqrt{\phi_{0}+3 / 2}}-\sqrt{r(\xi)+\frac{1}{2}}\right. \\
& \left.-\frac{1}{2 \sqrt{r(\xi)+1 / 2}}\right), \quad-\xi_{6}<\xi<+\infty,
\end{aligned}
$$

where $\bar{\omega}=(1 / 3) \sqrt{3 \omega}, \xi_{6}=(1 / \bar{\omega})\left(\sqrt{\phi_{0}+3 / 2}+1 / 2 \sqrt{\phi_{0}+3 / 2}-\right.$ $\sqrt{2}),-3 / 2<\phi_{0}<-1$.

Example 14. Taking $\omega=-1.2, \tau=0.2, \phi_{0}=-0.8$, we get the approximations of $\Omega, \bar{\Omega}, \phi_{c}, \phi_{2}, \xi_{1}$ in formula (37), where 


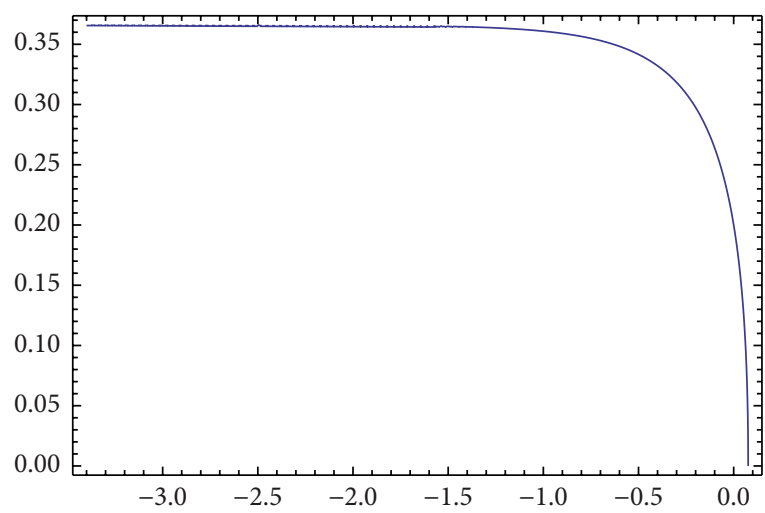

(a)

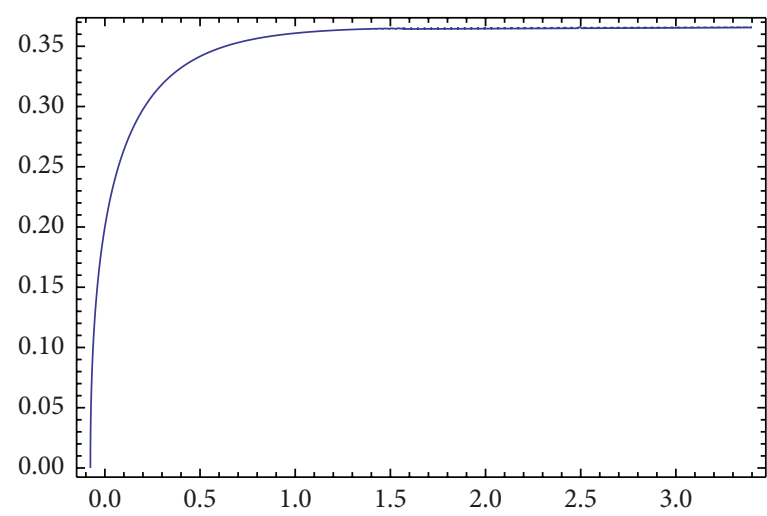

(b)

FIGURE 8: Kink-like waves of (3).

$\Omega \doteq 0.8, \bar{\Omega} \doteq 4.10263, \phi_{c} \doteq 0.8660254033, \phi_{2} \doteq$ $-0.633974596, \xi_{1} \doteq 0.0761185$. The profiles of (37) are shown in Figures 8(a) and 8(b), respectively.

\section{The Derivations to Main Results}

4.1. The Derivations for Proposition 1. For given $h \in\left(-\infty, h_{1}\right)$ in Figures 1(e), 1(f), and 1(g), respectively, the level curves are shown in Figures 9(a), 9(b), and 9(c), respectively. From Figures 9(a), 9(b), and 9(c), we see that there are two open curves of system (18) defined by $H(\phi, y)=h$ passing points $\left(\gamma_{1}, 0\right)$ and $\left(\gamma_{2}, 0\right)$, respectively, and approaching the line $\phi=$ -1 when $\omega<0,0<\tau<-(1 / 2) \omega$, and $h \in\left(-\infty, h_{1}\right)$, where $\gamma_{1}, \gamma_{2}, \gamma_{3}\left(\gamma_{3}<\gamma_{2}<\gamma_{1}\right)$ are three real roots of $(\omega+2 \tau) X^{3}+$ $(2 \omega+4 \tau+h) X^{2}+2(\omega+\tau+h) X+(\omega+\tau+h)=0$. Their expressions are

$$
y= \pm \frac{\sqrt{-(\omega+2 \tau)\left(\gamma_{1}-\phi\right)\left(\phi-\gamma_{2}\right)\left(\phi-\gamma_{3}\right)}}{1+\phi}
$$

$$
\gamma_{2} \leq \phi \leq \gamma_{1}, \phi \neq-1 .
$$

Substituting (43) into $d \phi / d \chi=(1+\phi) y$ and integrating it along the open curves yield equation

$$
\int_{\phi}^{\gamma_{1}} \frac{d s}{\sqrt{\left(\gamma_{1}-s\right)\left(s-\gamma_{2}\right)\left(s-\gamma_{3}\right)}}=\sqrt{-(\omega+2 \tau)}|\chi| .
$$

Completing (44) and using transformations $d \xi=(1+\phi) d \chi$ and $r(\xi)=1+\phi(\xi)$, we can get a periodic loop soliton solution of (3) same as (23).

For given $h \in(-\infty, 0)$ in Figure 1(h), the level curve is shown in Figure 9(d). From Figure 9(d), we see that there are two open curves of system (18) defined by $H(\phi, y)=h$ passing points $\left(\gamma_{1}, 0\right)$ and $\left(\gamma_{2}, 0\right)$, respectively, and approaching the line $\phi=-1$ when $\omega<0, \tau=-(1 / 2) \omega$, and $h \in(-\infty, 0)$, where $\gamma_{1,2}=-(\omega+2 h \pm \sqrt{\omega(\omega+2 h)}) / 2 h$. Their expressions are

$$
y= \pm \frac{\sqrt{-h\left(\gamma_{1}-\phi\right)\left(\phi-\gamma_{2}\right)}}{1+\phi}, \quad \gamma_{2} \leq \phi \leq \gamma_{1}, \phi \neq-1
$$

Substituting (45) into $d \phi / d \chi=(1+\phi) y$ and integrating it along the open curves yield equation

$$
\int_{\phi}^{\gamma_{1}} \frac{d s}{\sqrt{\left(\gamma_{1}-s\right)\left(s-\gamma_{2}\right)}}=\sqrt{-h}|\chi|
$$

Completing (46) and using transformations $d \xi=(1+\phi) d \chi$ and $r(\xi)=1+\phi(\xi)$, we can get a periodic loop soliton solution of (3) same as (24).

For given $h \in\left(-\infty, h_{0}\right)$ in Figures 1(i), 1(p), 1(q), and 1(r), respectively, the level curves are shown in Figures 9(e), 9(f), 9(g), and 9(h), respectively. From Figures 9(e), 9(f), 9(g), and $9(\mathrm{~h})$, we see that there are two open curves of system (18) defined by $H(\phi, y)=h$ passing points $\left(\gamma_{2}, 0\right)$ and $\left(\gamma_{3}, 0\right)$, respectively, and approaching the line $\phi=-1$ when $\omega<$ $0, \tau>-(1 / 2) \omega$ (or $\omega>0, \tau>0)$, and $h \in\left(-\infty, h_{0}\right)$, where $\gamma_{1}, \gamma_{2}, \gamma_{3}\left(\gamma_{3}<\gamma_{2}<\gamma_{1}\right)$ are three real roots of $(\omega+2 \tau) X^{3}+$ $(2 \omega+4 \tau+h) X^{2}+2(\omega+\tau+h) X+(\omega+\tau+h)=0$. Their expressions are

$$
y= \pm \frac{\sqrt{(\omega+2 \tau)\left(\gamma_{1}-\phi\right)\left(\gamma_{2}-\phi\right)\left(\phi-\gamma_{3}\right)}}{1+\phi}
$$

$$
\gamma_{3} \leq \phi \leq \gamma_{2}, \phi \neq-1
$$

Substituting (47) into $d \phi / d \chi=(1+\phi) y$ and integrating it along the open curves yield equation

$$
\int_{\gamma_{3}}^{\phi} \frac{d s}{\sqrt{\left(\gamma_{1}-s\right)\left(\gamma_{2}-s\right)\left(s-\gamma_{3}\right)}}=\sqrt{\omega+2 \tau}|\chi| .
$$

Completing (48) and using transformations $d \xi=(1+\phi) d \chi$ and $r(\xi)=1+\phi(\xi)$, we can get a periodic loop soliton solution of (3) same as (25).

4.2. The Derivations for Proposition 3. For given $h \in$ $(-\infty, 3 \omega)$ in Figure 1(d), the level curve is shown in Figure 9(i). From Figure 9(i), we see that there is one open curve of system (18) defined by $H(\phi, y)=h$ passing point $\left(\gamma_{1}, 0\right)$ and approaching the line $\phi=-1$ when $\omega<0, \tau=0$, and 


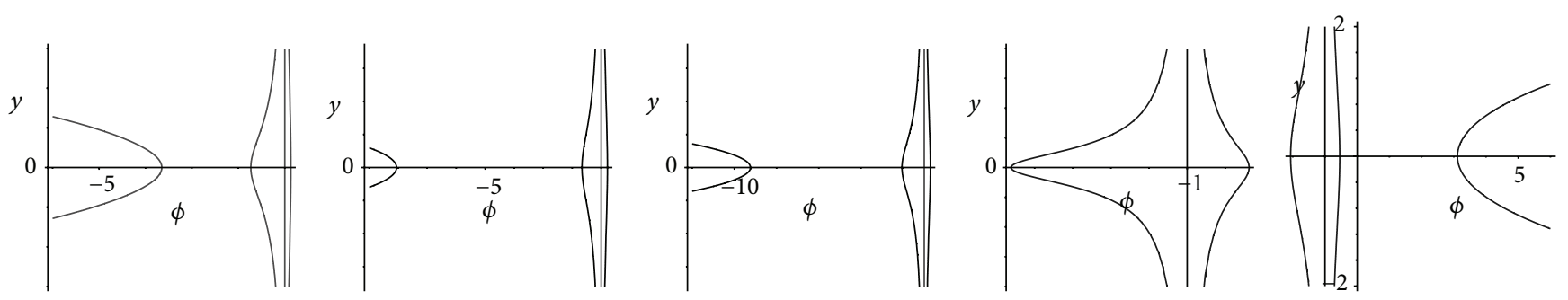

(a)

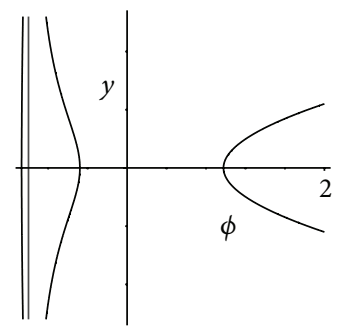

(f)

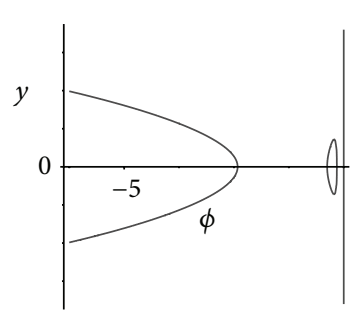

(k) (b)

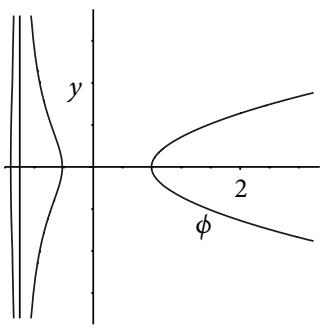

(g)

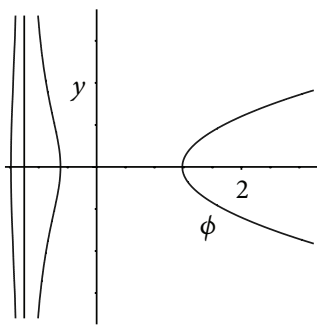

(h)

(d)

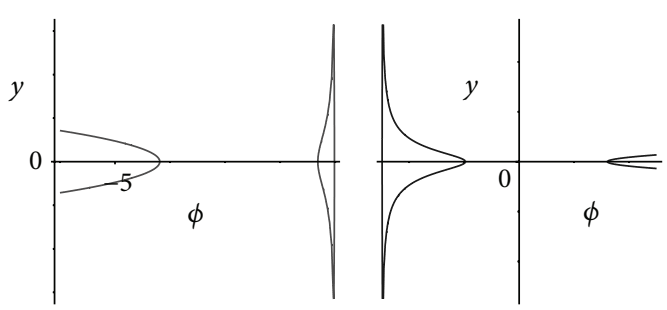

(i)

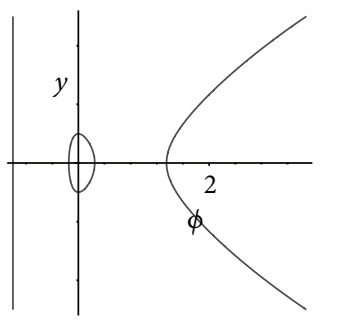

(n)

(j)

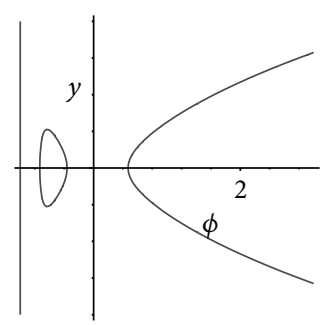

(o)

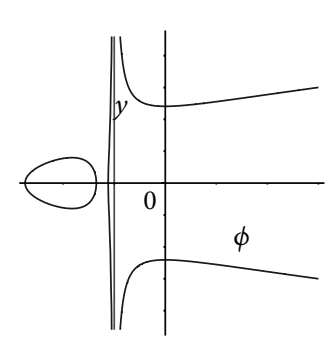

(p)

(1)

(m)

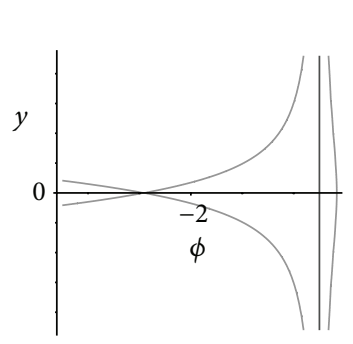

(q)

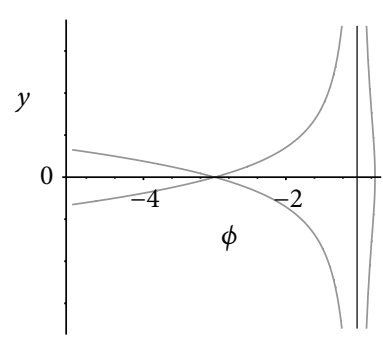

(r)

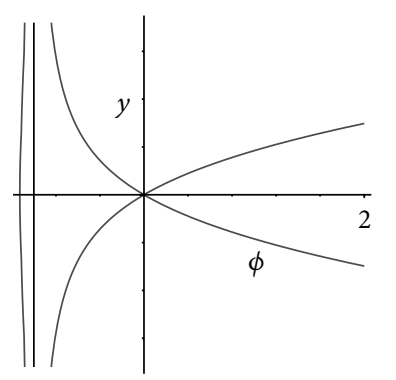

(v)

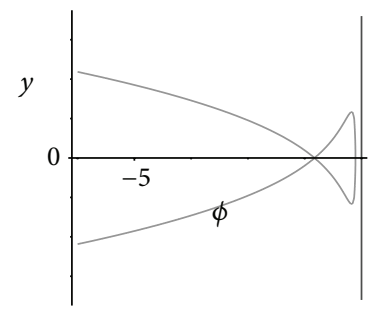

(z)

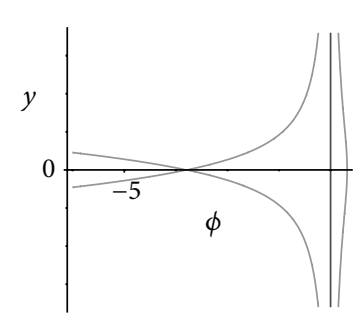

(s)

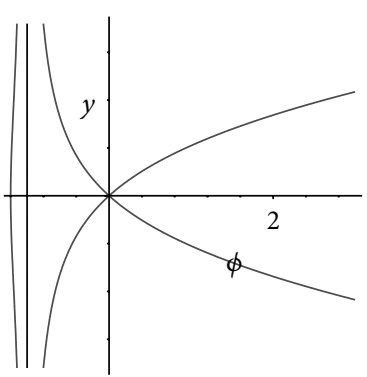

(w)

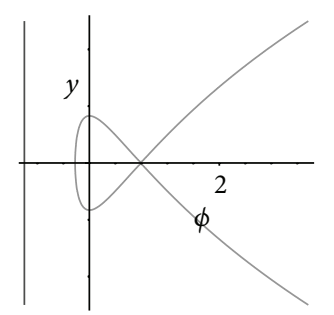

(aa)

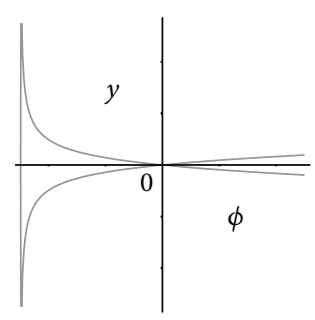

(y)

FIGUre 9: Continued. 


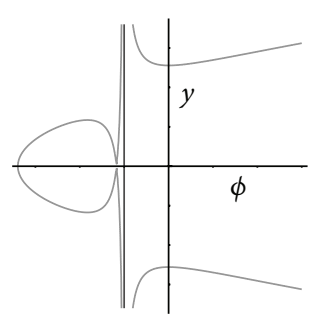

(ab)

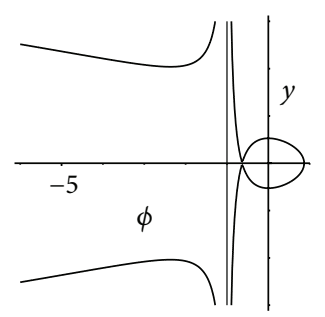

(ac)

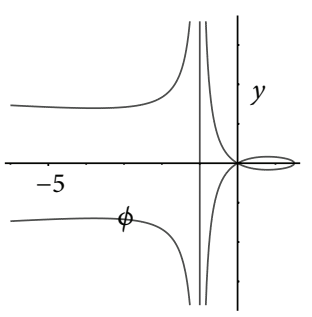

(ad)

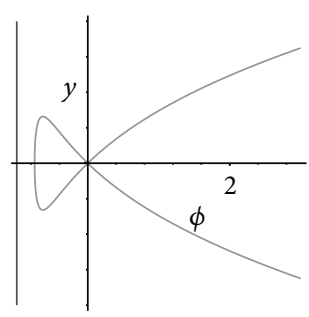

(ae)

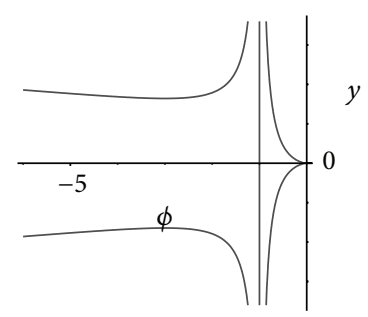

(af)

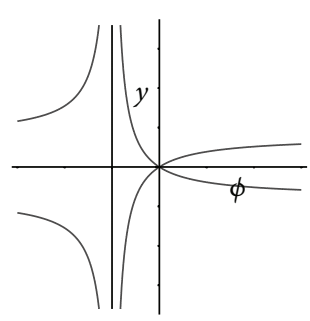

$(\mathrm{ag})$

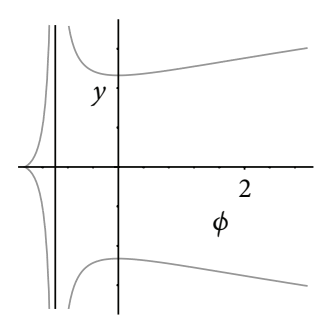

(ah)

Figure 9: The level curves defined by $H(\phi, y)=h$ of (18).

$h \in(-\infty, 3 \omega)$, where $\gamma_{1,2}=-(h+\omega \pm \sqrt{(h+\omega)(h-3 \omega)}) / 2 \omega$. Its expression is

$$
y= \pm \frac{\sqrt{-\omega(-1-\phi)\left(\phi-\gamma_{1}\right)\left(\phi-\gamma_{2}\right)}}{1+\phi}
$$

$$
\gamma_{1} \leq \phi<-1
$$

Substituting (49) into $d \phi / d \chi=(1+\phi) y$ and integrating it along the open curve yield equation

$$
\int_{\phi}^{-1} \frac{d s}{\sqrt{(-1-s)\left(s-\gamma_{1}\right)\left(s-\gamma_{2}\right)}}=\sqrt{-\omega}|\chi| \text {. }
$$

Completing (50) and using transformations $d \xi=(1+\phi) d \chi$ and $r(\xi)=1+\phi(\xi)$, we can get a periodic cusp wave solution of (3) same as (26).

For given $h \in(-\infty,-\omega)$ in Figure 1(o), the level curve is shown in Figure $9(j)$. From Figure $9(j)$, we see that there is one open curve of system (18) defined by $H(\phi, y)=h$ passing point $\left(\gamma_{2}, 0\right)$ and approaching the line $\phi=-1$ when $\omega>0, \tau=0$, and $h \in(-\infty,-\omega)$, where $\gamma_{1,2}=-(h+\omega \mp$ $\left.\sqrt{h^{2}-2 \omega h-3 \omega^{2}}\right) / 2 \omega$. Its expression is

$$
y= \pm \frac{\sqrt{\omega\left(\gamma_{1}-\phi\right)\left(\gamma_{2}-\phi\right)(\phi+1)}}{1+\phi}, \quad-1<\phi \leq \gamma_{2} .
$$

Substituting (51) into $d \phi / d \chi=(1+\phi) y$ and integrating it along the open curve yield equation

$$
\int_{-1}^{\phi} \frac{d s}{\sqrt{\left(\gamma_{1}-s\right)\left(\gamma_{2}-s\right)(s+1)}}=\sqrt{\omega}|\chi| .
$$

Completing (52) and using transformations $d \xi=(1+\phi) d \chi$ and $r(\xi)=1+\phi(\xi)$, we can get a periodic cusp wave solution of (3) same as (27).
4.3. The Derivations for Proposition 5. For given $h \in\left(h_{2}, h_{1}\right)$ in Figure $1(\mathrm{c}), h \in\left(h_{0}, h_{2}\right)$ in Figure 1(e), and $h \in\left(h_{2}, h_{0}\right)$ in Figure $1(\mathrm{~g})$, respectively, the level curves are shown in Figures 9(k), 9(l), and 9(m), respectively. From Figures 9(k), 9(l), and $9(\mathrm{~m})$, we see that there is one periodic orbit of system (18) defined by $H(\phi, y)=h$ passing points $\left(\gamma_{1}, 0\right)$ and $\left(\gamma_{2}, 0\right)$ when $\omega<0,(1 / 6) \omega<\tau<0, h \in\left(h_{2}, h_{1}\right)$ (or $\omega<0,0<\tau<$ $-(1 / 3) \omega, h \in\left(h_{0}, h_{2}\right)$ or $\omega<0,-(1 / 3) \omega<\tau<-(1 / 2) \omega, h \in$ $\left.\left(h_{2}, h_{0}\right)\right)$, where $\gamma_{1}, \gamma_{2}, \gamma_{3}\left(\gamma_{3}<\gamma_{2}<\gamma_{1}\right)$ are three real roots of $(\omega+2 \tau) X^{3}+(2 \omega+4 \tau+h) X^{2}+2(\omega+\tau+h) X+(\omega+\tau+h)=0$. Its expression is

$$
\begin{aligned}
& y= \pm \frac{\sqrt{-(\omega+2 \tau)\left(\gamma_{1}-\phi\right)\left(\phi-\gamma_{2}\right)\left(\phi-\gamma_{3}\right)}}{1+\phi} \\
& \gamma_{2} \leq \phi \leq \gamma_{1} .
\end{aligned}
$$

Substituting (53) into $d \phi / d \chi=(1+\phi) y$ and integrating it along the periodic orbit yield equation

$$
\int_{\phi}^{\gamma_{1}} \frac{d s}{\sqrt{\left(\gamma_{1}-s\right)\left(s-\gamma_{2}\right)\left(s-\gamma_{3}\right)}}=\sqrt{-(\omega+2 \tau)}|\chi| .
$$

Completing (54) and using transformations $d \xi=(1+\phi) d \chi$ and $r(\xi)=1+\phi(\xi)$, we can get a smooth periodic wave solution of (3) same as (28).

For given $h \in\left(h_{0}, h_{1}\right)$ in Figure $1(1), h \in\left(h_{1}, h_{0}\right)$ in Figure $1(\mathrm{n})$, and $h \in\left(h_{2}, h_{1}\right)$ in Figure $1(\mathrm{p})$, respectively, the level curves are shown in Figures 9(n), 9(o), and 9(p), respectively. From Figures 9(n), 9(o), and 9(p), we see that there is one periodic orbit of system (18) defined by $H(\phi, y)=$ $h$ passing points $\left(\gamma_{2}, 0\right)$ and $\left(\gamma_{3}, 0\right)$ when $\omega>0,-(1 / 2) \omega<$ $\tau<-(1 / 3) \omega, h \in\left(h_{0}, h_{1}\right)($ or $\omega>0,-(1 / 3) \omega<\tau<0$, $h \in\left(h_{1}, h_{0}\right)$ or $\left.\omega>0,0<\tau<(1 / 6) \omega, h \in\left(h_{2}, h_{1}\right)\right)$, where $\gamma_{1}, \gamma_{2}, \gamma_{3}\left(\gamma_{3}<\gamma_{2}<\gamma_{1}\right)$ are three real roots of 
$(\omega+2 \tau) X^{3}+(2 \omega+4 \tau+h) X^{2}+2(\omega+\tau+h) X+(\omega+\tau+h)=0$. Its expression is

$$
y= \pm \frac{\sqrt{(\omega+2 \tau)\left(\gamma_{1}-\phi\right)\left(\gamma_{2}-\phi\right)\left(\phi-\gamma_{3}\right)}}{1+\phi}
$$

$$
\gamma_{3} \leq \phi \leq \gamma_{2}
$$

Substituting (55) into $d \phi / d \chi=(1+\phi) y$ and integrating it along the periodic orbit yield equation

$$
\int_{\gamma_{3}}^{\phi} \frac{d s}{\sqrt{\left(\gamma_{1}-s\right)\left(\gamma_{2}-s\right)\left(s-\gamma_{3}\right)}}=\sqrt{\omega+2 \tau}|\chi| .
$$

Completing (56) and using transformations $d \xi=(1+\phi) d \chi$ and $r(\xi)=1+\phi(\xi)$, we can get a smooth periodic wave solution of (3) same as (29).

4.4. The Derivations for Proposition 7. For given $h=h_{1}$ in Figures 1(e), 1(f), and 1(g), respectively, the level curves are shown in Figures 9(q), 9(r), and 9(s), respectively. From Figures $9(\mathrm{q}), 9(\mathrm{r})$, and $9(\mathrm{~s})$, we see that there are three open curves of system (18) defined by $H(\phi, y)=h_{1}$, one of them passing point $\left(\phi_{a}, 0\right)$ and approaching the line $\phi=-1$ and the others both passing saddle point $\left(\phi_{1}, 0\right)$ and approaching the line $\phi=-1$ when $\omega<0,0<\tau<-(1 / 2) \omega$, where $\phi_{a}=-(\sqrt{\Delta}-\omega) /(\sqrt{\Delta}-\omega+2 \tau)$. Their expressions are

$$
y= \pm \frac{\left(\phi-\phi_{1}\right) \sqrt{\Omega\left(\phi_{a}-\phi\right)}}{1+\phi}, \quad \phi_{1}<\phi \leq \phi_{a}, \phi \neq-1,
$$

where $\Omega=2(\omega+2 \tau)^{2}(\sqrt{\Delta}-(\omega-2 \tau)) /(\sqrt{\Delta}-(\omega+2 \tau))^{2}$. Substituting (57) into $d \phi / d \xi=y$ and integrating it along the open curves yield equation

$$
\int_{\phi}^{\phi_{a}} \frac{(1+s) d s}{\left(s-\phi_{1}\right) \sqrt{\phi_{a}-s}}=\sqrt{\Omega}|\xi| .
$$

Completing (58) and using transformation $r(\xi)=1+\phi(\xi)$, we can get a loop soliton solution of (3) same as (30).

For given $h=h_{0}$ in Figures 1(i), 1(p), 1(q), and 1(r), respectively, the level curves are shown in Figures $9(\mathrm{t}), 9(\mathrm{u})$, 9(v), and 9(w), respectively. From Figures 9(t), 9(u), 9(v), and $9(\mathrm{w})$, we see that there are three open curves of system (18) defined by $H(\phi, y)=h_{0}$, one of them passing point $\left(\phi_{b}, 0\right)$ and approaching the line $\phi=-1$ and the others both passing saddle point $(0,0)$ and approaching the line $\phi=-1$ when $\omega<0, \tau>-(1 / 2) \omega$ (or $\omega>0, \tau>0$ ), where $\phi_{b}=-(\omega+3 \tau) /(\omega+2 \tau)$. Their expressions are

$$
y= \pm \frac{\phi \sqrt{(\omega+2 \tau)\left(\phi-\phi_{b}\right)}}{1+\phi}, \quad \phi_{b} \leq \phi<0, \phi \neq-1 .
$$

Substituting (59) into $d \phi / d \chi=(1+\phi) y$ and integrating it along the open curves yield equation

$$
\int_{\phi_{b}}^{\phi} \frac{d s}{s \sqrt{s-\phi_{b}}}=-\sqrt{\omega+2 \tau}|\chi| \text {. }
$$

Completing (60) and using transformations $d \xi=(1+\phi) d \chi$ and $r(\xi)=1+\phi(\xi)$, we can get a loop soliton solution of (3) same as (31).

4.5. The Derivations for Proposition 9. For given $h=3 \omega$ in Figure 1(d), the level curve is shown in Figure 9(x). From Figure $9(\mathrm{x})$, we see that there are two open curves of system (18) defined by $H(\phi, y)=3 \omega$ both passing saddle point $(-2,0)$ and approaching the line $\phi=-1$ when $\omega<0, \tau=0$. Their expressions are

$$
y= \pm \frac{(\phi+2) \sqrt{-\omega(-1-\phi)}}{1+\phi}, \quad-2<\phi<-1 .
$$

Substituting (61) into $d \phi / d \chi=(1+\phi) y$ and integrating it along the open curves yield equation

$$
\int_{\phi}^{-1} \frac{d s}{(s+2) \sqrt{-1-s}}=\sqrt{-\omega}|\chi| .
$$

Completing (62) and using transformations $d \xi=(1+\phi) d \chi$ and $r(\xi)=1+\phi(\xi)$, we can get a cuspon solution of (3) same as (32).

For given $h=-\omega$ in Figure 1(o), the level curve is shown in Figure 9(y). From Figure 9(y), we see that there are two open curves of system (18) defined by $H(\phi, y)=-\omega$ both passing saddle point $(0,0)$ and approaching the line $\phi=-1$ when $\omega>0, \tau=0$. Their expressions are

$$
y= \pm \frac{\phi \sqrt{\omega(\phi+1)}}{1+\phi}, \quad-1<\phi<0 .
$$

Substituting (63) into $d \phi / d \chi=(1+\phi) y$ and integrating it along the open curves yield equation

$$
\int_{-1}^{\phi} \frac{d s}{s \sqrt{s+1}}=\sqrt{\omega}|\chi| .
$$

Completing (64) and using transformations $d \xi=(1+\phi) d \chi$ and $r(\xi)=1+\phi(\xi)$, we can get a cuspon solution of (3) same as (33).

4.6. The Derivations for Proposition 11. For given $h=h_{1}$ in Figures 1(c), 1(l), and 1(p), respectively, the level curves are shown in Figures $9(\mathrm{z}), 9(\mathrm{aa})$ and $9(\mathrm{ab})$, respectively. From Figures 9(z), 9(aa) and 9(ab), we see that there is a homoclinic orbit of system (18) defined by $H(\phi, y)=h_{1}$ connecting with the saddle point $\left(\phi_{1}, 0\right)$ and passing point $\left(\phi_{a}, 0\right)$ when $\omega<0,(1 / 6) \omega<\tau<0$ (or $\omega>0,-(1 / 2) \omega<\tau<-(1 / 3) \omega$ or $\omega>0,0<\tau<(1 / 6) \omega)$, where $\phi_{a}$ is given in (30). When $\omega<0,(1 / 6) \omega<\tau<0$, its expression is

$$
y= \pm \frac{\left(\phi-\phi_{1}\right) \sqrt{\Omega\left(\phi_{a}-\phi\right)}}{1+\phi}, \quad \phi_{1}<\phi \leq \phi_{a}
$$

and when $\omega>0,-(1 / 2) \omega<\tau<-(1 / 3) \omega$ (or $\omega>0,0<$ $\tau<(1 / 6) \omega)$, its expression is

$$
y= \pm \frac{\left(\phi_{1}-\phi\right) \sqrt{\Omega\left(\phi-\phi_{a}\right)}}{1+\phi}, \quad \phi_{a} \leq \phi<\phi_{1},
$$


where $\Omega$ is given in (30). Substituting (65) into $d \phi / d \chi=$ $(1+\phi) y$ and integrating it along the homoclinic orbit yield equation

$$
\int_{\phi}^{\phi_{a}} \frac{d s}{\left(s-\phi_{1}\right) \sqrt{\phi_{a}-s}}=\sqrt{\Omega}|\chi| .
$$

Completing (67) and using transformations $d \xi=(1+\phi) d \chi$ and $r(\xi)=1+\phi(\xi)$, we can get a smooth solitary wave solution of (3) same as (34).

Substituting (66) into $d \phi / d \chi=(1+\phi) y$ and integrating it along the homoclinic orbit yield equation

$$
\int_{\phi_{a}}^{\phi} \frac{d s}{\left(\phi_{1}-s\right) \sqrt{s-\phi_{a}}}=\sqrt{\Omega}|\chi| .
$$

Completing (68) and using transformations $d \xi=(1+\phi) d \chi$ and $r(\xi)=1+\phi(\xi)$, we also can get a smooth solitary wave solution of (3) same as (34).

For given $h=h_{2}$ in Figure 1(e), the level curve is shown in Figure 9(ac). From Figure 9(ac), we see that there is a homoclinic orbit of system (18) defined by $H(\phi, y)=h_{2}$ connecting with the saddle point $\left(\phi_{2}, 0\right)$ and passing point $\left(\phi_{c}, 0\right)$ when $\omega<0,0<\tau<-(1 / 3) \omega$, where $\phi_{c}$ is given in (35). Its expression is

$$
y= \pm \frac{\left(\phi-\phi_{2}\right) \sqrt{\Omega\left(\phi_{c}-\phi\right)}}{1+\phi}, \quad \phi_{2}<\phi \leq \phi_{c}
$$

where $\Omega$ is given in (35). Substituting (69) into $d \phi / d \chi=$ $(1+\phi) y$ and integrating it along the homoclinic orbit yield equation

$$
\int_{\phi}^{\phi_{c}} \frac{d s}{\left(s-\phi_{2}\right) \sqrt{\phi_{c}-s}}=\sqrt{\Omega}|\chi| .
$$

Completing (70) and using transformations $d \xi=(1+\phi) d \chi$ and $r(\xi)=1+\phi(\xi)$, we can get a smooth solitary wave solution of (3) same as (35).

For given $h=h_{0}$ in Figures $1(\mathrm{~g})$ and 1(n), respectively, the level curves are shown in Figures $9(\mathrm{ad})$ and $9(\mathrm{ae})$, respectively. From Figures 9(ad) and 9(ae), we see that there is a homoclinic orbit of system (18) defined by $H(\phi, y)=h_{0}$ connecting with the saddle point $(0,0)$ and passing point $\left(\phi_{b}, 0\right)$ when $\omega<0,-(1 / 3) \omega<\tau<-(1 / 2) \omega$ (or $\omega>$ $0,-(1 / 3) \omega<\tau<0)$, where $\phi_{b}$ is given in (31). When $\omega<0,-(1 / 3) \omega<\tau<-(1 / 2) \omega$, its expression is

$$
y= \pm \frac{\phi \sqrt{-(\omega+2 \tau)\left(\phi_{b}-\phi\right)}}{1+\phi}, \quad 0<\phi \leq \phi_{b} \text {, }
$$

and when $\omega>0,-(1 / 3) \omega<\tau<0$, its expression is

$$
y= \pm \frac{\phi \sqrt{(\omega+2 \tau)\left(\phi-\phi_{b}\right)}}{1+\phi}, \quad \phi_{b} \leq \phi<0 .
$$

Substituting (71) into $d \phi / d \chi=(1+\phi) y$ and integrating it along the homoclinic orbit yield equation

$$
\int_{\phi}^{\phi_{b}} \frac{d s}{s \sqrt{\phi_{b}-s}}=\sqrt{-(\omega+2 \tau)}|\chi| .
$$

Completing (73) and using transformations $d \xi=(1+\phi) d \chi$ and $r(\xi)=1+\phi(\xi)$, we can get a smooth solitary wave solution of (3) same as (36).

Substituting (72) into $d \phi / d \chi=(1+\phi) y$ and integrating it along the homoclinic orbit yield equation

$$
\int_{\phi_{b}}^{\phi} \frac{d s}{s \sqrt{s-\phi_{b}}}=\sqrt{(\omega+2 \tau)}|\chi| \text {. }
$$

Completing (74) and using transformations $d \xi=(1+\phi) d \chi$ and $r(\xi)=1+\phi(\xi)$, we also can get a smooth solitary wave solution of (3) same as (36).

4.7. The Derivations for Proposition 13. From Figure 9(ac), we see that there are two open curves of system (18) defined by $H(\phi, y)=h_{2}$ both passing saddle point $\left(\phi_{2}, 0\right)$ and approaching the line $\phi=-1$ when $\omega<0,0<\tau<-(1 / 3) \omega$. Their expressions are

$$
y= \pm \frac{\left(\phi_{2}-\phi\right) \sqrt{\Omega\left(\phi_{c}-\phi\right)}}{1+\phi}, \quad-1<\phi<\phi_{2}
$$

where $\phi_{c}, \Omega$ are given in (35). Substituting (75) into $d \phi / d \xi=$ $y$ and integrating it along the open curves yield equation

$$
\int_{\phi}^{\phi_{0}} \frac{(1+s) d s}{\left(\phi_{2}-s\right) \sqrt{\phi_{c}-s}}= \pm \sqrt{\Omega} \xi
$$

where $-1<\phi_{0}<\phi_{2}$. Completing (76) and using transformation $r(\xi)=1+\phi(\xi)$, we can get two kink-like wave solutions of implicit expression of (3) same as (37).

For given $h=-(2 / 3) \omega$ in Figure 1(f), the level curve is shown in Figure 9(af). From Figure 9(af), we see that there are two open curves of system (18) defined by $H(\phi, y)=-(2 / 3) \omega$ both passing saddle point $(0,0)$ and approaching the line $\phi=$ -1 when $\omega<0, \tau=-(1 / 3) \omega$. Their expressions are

$$
y= \pm \frac{\phi \sqrt{3 \omega \phi}}{3(1+\phi)}, \quad-1<\phi<0 .
$$

Substituting (77) into $d \phi / d \xi=y$ and integrating it along the open curves yield equation

$$
\int_{\phi}^{\phi_{0}} \frac{(1+s) d s}{s \sqrt{-s}}= \pm \frac{1}{3} \sqrt{-3 \omega} \xi
$$

where $-1<\phi_{0}<0$. Completing (78) and using transformation $r(\xi)=1+\phi(\xi)$, we can get two kink-like wave solutions of implicit expression of (3) same as (38).

From Figure $9(\mathrm{ad})$, we see that there are two open curves of system (18) defined by $H(\phi, y)=h_{0}$ both passing saddle point $(0,0)$ and approaching the line $\phi=-1$ when $\omega<$ $0,-(1 / 3) \omega<\tau<-(1 / 2) \omega$. Their expressions are

$$
y= \pm \frac{\phi \sqrt{-\omega\left(\phi_{b}-\phi\right)}}{1+\phi}, \quad-1<\phi<0
$$


where $\phi_{b}$ is given in (31). Substituting (79) into $d \phi / d \xi=y$ and integrating it along the open curves yield equation

$$
\int_{\phi}^{\phi_{0}} \frac{(1+s) d s}{s \sqrt{\phi_{b}-s}}= \pm \sqrt{-\omega} \xi
$$

where $-1<\phi_{0}<0$. Completing (80) and using transformation $r(\xi)=1+\phi(\xi)$, we can get two kink-like wave solutions of implicit expression of (3) same as (39).

For given $h=-(1 / 2) \omega$ in Figure $1(\mathrm{~h})$, the level curve is shown in Figure 9(ag). From Figure 9(ag), we see that there are two open curves of system (18) defined by $H(\phi, y)=$ $-(1 / 2) \omega$ both passing saddle point $(0,0)$ and approaching the line $\phi=-1$ when $\omega<0, \tau=-(1 / 2) \omega$. Their expressions are

$$
y= \pm \frac{\sqrt{-2 \omega}}{2} \frac{\phi}{1+\phi}, \quad-1<\phi<0 .
$$

Substituting (81) into $d \phi / d \xi=y$ and integrating it along the open curves yield equation

$$
\int_{\phi}^{\phi_{0}} \frac{(1+s) d s}{s}= \pm \frac{1}{2} \sqrt{-2 \omega} \xi
$$

where $-1<\phi_{0}<0$. Completing (82) and using transformation $r(\xi)=1+\phi(\xi)$, we can get two kink-like wave solutions of implicit expression of (3) same as (40).

From Figure 9(ab), we see that there are two open curves of system (18) defined by $H(\phi, y)=h_{1}$ both passing saddle point $\left(\phi_{1}, 0\right)$ and approaching the line $\phi=-1$ when $\omega>$ $0,0<\tau<(1 / 6) \omega$. Their expressions are

$$
y= \pm \frac{\left(\phi-\phi_{1}\right) \sqrt{\Omega\left(\phi-\phi_{a}\right)}}{1+\phi}, \quad \phi_{1}<\phi<-1,
$$

where $\phi_{a}, \Omega$ are given in (30). Substituting (83) into $d \phi / d \xi=$ $y$ and integrating it along the open curves yield equation

$$
\int_{\phi}^{\phi_{0}} \frac{(1+s) d s}{\left(s-\phi_{1}\right) \sqrt{s-\phi_{a}}}= \pm \sqrt{\Omega} \xi
$$

where $\phi_{1}<\phi_{0}<-1$. Completing (84) and using transformation $r(\xi)=1+\phi(\xi)$, we can get two kink-like wave solutions of implicit expression of (3) same as (41).

For given $h=(10 / 3) \omega$ in Figure 1(q), the level curve is shown in Figure 9(ah). From Figure 9(ah), we see that there are two open curves of system (18) defined by $H(\phi, y)=$ $(10 / 3) \omega$ both passing saddle point $(-3 / 2,0)$ and approaching the line $\phi=-1$ when $\omega>0, \tau=(1 / 6) \omega$. Their expressions are

$$
y= \pm \frac{2(\phi+3 / 2) \sqrt{3 \omega(\phi+3 / 2)}}{3(1+\phi)}, \quad-\frac{3}{2}<\phi<-1 .
$$

Substituting (85) into $d \phi / d \xi=y$ and integrating it along the open curves yield equation

$$
\int_{\phi}^{\phi_{0}} \frac{(1+s) d s}{(s+3 / 2) \sqrt{s+3 / 2}}= \pm \frac{2}{3} \sqrt{3 \omega} \xi
$$

where $-3 / 2<\phi_{0}<-1$. Completing (86) and using transformation $r(\xi)=1+\phi(\xi)$, we can get two kink-like wave solutions of implicit expression of (3) same as (42).

The derivations for our main results are completed.

\section{Remarks and Conclusion}

\subsection{Some Remarks}

Remark 15. From Figures 1(e), 1(f), and 1(g), we see that the two open curves approach the three open curves gradually when $\omega<0,0<\tau<-(1 / 2) \omega$, and $h \rightarrow h_{1}$. Therefore, the periodic loop soliton solution (23) converges to the loop soliton solution (30) as $h$ approaches $h_{1}$.

Remark 16. From Figures 1(i), 1(p), 1(q), and 1(r), we see that the two open curves approach the three open curves gradually when $\omega<0, \tau>-(1 / 2) \omega$ (or $\omega>0, \tau>0$ ), and $h \rightarrow h_{0}$. Therefore, the periodic loop soliton solution (25) converges to the loop soliton solution (31) as $h$ approaches $h_{0}$.

Remark 17. From Figure 1(d), we see that the one open curve approaches the two open curves gradually when $\omega<0, \tau=$ 0 , and $h \rightarrow 3 \omega$. Therefore, the periodic cusp wave solution (26) converges to the cuspon solution (32) as $h$ approaches $3 \omega$.

Remark 18. From Figure 1(o), we see that the one open curve approaches the two open curves gradually when $\omega>0, \tau=$ 0 , and $h \rightarrow-\omega$. Therefore, the periodic cusp wave solution (27) converges to the cuspon solution (33) as $h$ approaches $-\omega$.

Remark 19. From Figure 1(c), we see that the periodic orbit approaches the homoclinic orbit gradually when $\omega<$ 0 , $(1 / 6) \omega<\tau<0$, and $h \rightarrow h_{1}$. Therefore, the smooth periodic wave solution (28) converges to the smooth solitary solution (34) as $h$ approaches $h_{1}$.

Remark 20. From Figure 1(e), we see that the periodic orbit approaches the homoclinic orbit gradually when $\omega<0,0<$ $\tau<-(1 / 3) \omega$, and $h \rightarrow h_{2}$. Therefore, the smooth periodic wave solution (28) converges to the smooth solitary solution (35) as $h$ approaches $h_{2}$.

Remark 21. From Figure $1(\mathrm{~g})$, we see that the periodic orbit approaches the homoclinic orbit gradually when $\omega<$ $0,-(1 / 3) \omega<\tau<-(1 / 2) \omega$, and $h \rightarrow h_{0}$. Therefore, the smooth periodic wave solution (28) converges to the smooth solitary solution (36) as $h$ approaches $h_{0}$.

Remark 22. From Figures 1(l) and 1(p), we see that the periodic orbit approaches the homoclinic orbit gradually when $\omega>0,-(1 / 2) \omega<\tau<-(1 / 3) \omega$ (or $\omega>0,0<\tau<$ $(1 / 6) \omega)$, and $h \rightarrow h_{1}$. Therefore, the smooth periodic wave solution (29) converges to the smooth solitary solution (34) as $h$ approaches $h_{1}$.

Remark 23. From Figure 1(n), we see that the periodic orbit approaches the homoclinic orbit gradually when $\omega>$ $0,-(1 / 3) \omega<\tau<0$, and $h \rightarrow h_{0}$. Therefore, the smooth periodic wave solution (29) converges to the smooth solitary solution (36) as $h$ approaches $h_{0}$. 
Remark 24. Geng et al. [26] indicated that the traveling wave solutions of (2) can be obtained from

$$
\gamma_{\xi}^{2}=\left(\mu \gamma^{3}+\nu \gamma^{2}+\omega \gamma+\tau\right) \gamma^{-2}
$$

and listed some traveling wave solutions (see (4)-(8) and (10)-(13)). In fact, some other traveling wave solutions also can be presented from (87) by choosing the parameters $\mu, \nu, \omega$, and $\tau$. For example, if $\mu=1, \nu=-2, \omega=-1, \tau=2$, then (87) yields equation

$$
\frac{d \gamma}{d \xi}=\gamma^{-1} \sqrt{(\gamma-2)(\gamma-1)(\gamma+1)}
$$

Let $d \xi=\gamma d s$, and we have

$$
\frac{d \gamma}{d s}=\sqrt{(\gamma-2)(\gamma-1)(\gamma+1)} \text {. }
$$

From (89) and $d \xi=\gamma d s$, we can present traveling wave solutions of (2) as follows:

$$
\begin{aligned}
& r(\xi)=2 \operatorname{sn}^{2}\left(\frac{\sqrt{3}}{2} s, \frac{\sqrt{6}}{3}\right)-1, \\
& \xi=2 s-2 \sqrt{3} E\left(\operatorname{am}\left(\frac{\sqrt{3}}{2} s, \frac{\sqrt{6}}{3}\right), \frac{\sqrt{6}}{3}\right), \\
& r(\xi)=\mathrm{nc}^{2}\left(\frac{\sqrt{3}}{2} s, \frac{\sqrt{6}}{3}\right)+1, \\
& \xi=2 s-2 \sqrt{3}\left(E\left(\operatorname{am}\left(\frac{\sqrt{3}}{2} s, \frac{\sqrt{6}}{3}\right), \frac{\sqrt{6}}{3}\right)\right. \\
& \left.-\operatorname{dn}\left(\frac{\sqrt{3}}{2} s, \frac{\sqrt{6}}{3}\right) \operatorname{tn}\left(\frac{\sqrt{3}}{2} s, \frac{\sqrt{6}}{3}\right)\right),
\end{aligned}
$$

where $\operatorname{sn}(\cdot, k), \mathrm{nc}(\cdot, k), \operatorname{dn}(\cdot, k), \operatorname{tn}(\cdot, k)$ are the Jacobian elliptic functions with the modulus $k$ [35]. However, to our knowledge, it is difficult to obtain the kink-like wave solutions of (2) from (87).

5.2. Conclusion. In this paper, we investigate the dynamic properties of the generalized HD type equation (2) and give some new bounded traveling wave solutions which include periodic loop soliton solutions, periodic cusp wave solutions, smooth periodic wave solutions, loop soliton solutions, cuspon solutions, smooth solitary wave solutions, and kink-like wave solutions in Propositions 1, 3, 5, 7, 9, 11 , and 13. Also, the relations of the periodic traveling wave solutions and other bounded traveling wave solutions are stated. Comparing with [26], we obtained some new results of (2). For example, we found some new periodic loop soliton, loop soliton, and kink-like wave solutions as (23)(25), (30) and (31), and (37)-(42), respectively. Obviously, the solutions which have been presented in this paper and [26] almost are exact implicit traveling wave solutions. Actually, there are some interesting and important problems to be further studied, for example, how to search for more new exact explicit traveling wave solutions and nontraveling wave solutions of (2). We will study (2) further.

\section{Competing Interests}

The authors declare that there are no competing interests regarding the publication of this paper.

\section{Acknowledgments}

This work is supported by the National Natural Science Foundation of China under Grant no. 11461022 and the Natural Science Foundations of Yunnan Province, China, under nos. 2014FA037 and 2013FZ117.

\section{References}

[1] C. Rogers and W. R. Shadwick, Bäcklund Transformation and Their Applications, Academic, New York, NY, USA, 1982.

[2] C. H. Gu, H. S. Hu, and Z. X. Zhou, Darboux Transformation in Soliton Theory and Its Geometric Applications, Shanghai Scientific and Technical Publishers, Shanghai, China, 1999.

[3] M. J. Ablowitz and P. A. Clarkson, Solitons, Nonlinear Evolution Equations and Inverse Scattering, Cambridge University Press, London, UK, 1991.

[4] R. Hirota, Direct Method in Soliton Theory, Springer, Berlin, Germany, 1980.

[5] H. Z. Liu and J. B. Li, "Painlevé analysis, complete Lie group classifications and exact solutions to the time-dependent coefficients Gardner types of equations," Nonlinear Dynamics, vol. 80, no. 1-2, pp. 515-527, 2015.

[6] W.-X. Ma and J.-H. Lee, "A transformed rational function method and exact solutions to the $3+1$ dimensional JimboMiwa equation," Chaos, Solitons \& Fractals, vol. 42, no. 3, pp. $1356-1363,2009$.

[7] H. Q. Zhang and W.-X. Ma, "Extended transformed rational function method and applications to complexiton solutions," Applied Mathematics and Computation, vol. 230, pp. 509-515, 2014.

[8] J.-H. He and X.-H. Wu, "Exp-function method for nonlinear wave equations," Chaos, Solitons \& Fractals, vol. 30, no. 3, pp. 700-708, 2006.

[9] W.-X. Ma and Z. Zhu, "Solving the $(3+1)$-dimensional generalized KP and BKP equations by the multiple exp-function algorithm," Applied Mathematics and Computation, vol. 218, no. 24, pp. 11871-11879, 2012.

[10] A. Biswas, A. H. Bhrawy, M. A. Abdelkawy, A. A. Alshaery, and E. M. Hilal, "Symbolic computation of some nonlinear fractional differential equations," Romanian Journal of Physics, vol. 59, no. 5-6, pp. 433-442, 2014.

[11] M. Kruskal, "Nonlinear wave equations," in Dynamical Systems, Theory and Applications, J. Moser, Ed., pp. 310-354, Springer, Berlin, Germany, 1975.

[12] M. Leo, R. A. Leo, G. Soliani, L. Solombrino, and L. Martina, "Lie-Bäcklund symmetries for the Harry-Dym equation," Physical Review D, vol. 27, no. 6, pp. 1406-1408, 1983.

[13] B. Fuchssteiner, T. Schulze, and S. Carillo, "Explicit solutions for the Harry Dym equation," Journal of Physics A: Mathematical and General, vol. 25, no. 1, pp. 223-230, 1992.

[14] B. Y. Guo and C. Rogers, "On the Harry Dym equation and its solution," Science China, vol. 32, pp. 283-295, 1989.

[15] C. Rogers and M. C. Nucci, "On reciprocal Bäcklund transformations and the Korteweg-de Vries hierarchy," Physica Scripta, vol. 33, no. 4, pp. 289-292, 1986. 
[16] S. Kawamoto, "An exact transformation from the Harry Dym equation to the modified KdV equation," Journal of the Physical Society of Japan, vol. 54, no. 5, pp. 2055-2056, 1985.

[17] M. Wadati, Y. H. Ichikawa, and T. Shimizu, "Cusp soliton of a new integrable nonlinear evolution equation," Progress of Theoretical Physics, vol. 64, no. 6, pp. 1959-1967, 1980.

[18] D. P. Novikov, "Algebraic-geometric solutions of the Harry Dym equation," Siberian Mathematical Journal, vol. 40, no. 1, pp. 136140, 1999.

[19] R. Mokhtari, "Exact solutions of the Harry-Dym equation," Communications in Theoretical Physics, vol. 55, no. 2, pp. 204208, 2011.

[20] V. G. Dubrovskyt and B. G. Konopelcbenkof, “ $\bar{\partial}$-dressing and exact solutions for the $(2+1)$-dimensional Harry Dym equation," Journal of Physics A: Mathematical and General, vol. 27, pp. 4619-4628, 1994.

[21] L. Dmitrieva and M. Khlabystova, "Multisoliton solutions of the (2+1)-dimensional Harry Dym equation," Physics Letters A, vol. 237, no. 6, pp. 369-380, 1998.

[22] M. Antonowicz and A. P. Fordy, "Coupled Harry Dym equations with multi-Hamiltonian structures," Journal of Physics A: Mathematical and General, vol. 21, no. 5, pp. L269-L275, 1988.

[23] J. B. Chen and X. G. Geng, "A new Neumann type integrable system related to the coupled Harry-Dym hierarchy," Physics Letters A, vol. 340, no. 1-4, pp. 181-187, 2005.

[24] W.-X. Ma, "An extended Harry Dym hierarchy," Journal of Physics A: Mathematical and Theoretical, vol. 43, no. 16, Article ID 165202, 13 pages, 2010.

[25] Z. Popowicz, "Two-component coupled KdV equations and its connection with the generalized Harry Dym equations," Journal of Mathematical Physics, vol. 55, no. 1, Article ID 013506, 8 pages, 2014.

[26] X. G. Geng, R. M. Li, and B. Xue, "A new integrable equation with peakons and cuspons and its bi-Hamiltonian structure," Applied Mathematics Letters, vol. 46, pp. 64-69, 2015.

[27] J. B. Li and H. H. Dai, On the Study of Singular Nonlinear Traveling Wave Equation: Dynamical System Approach, Science Press, Beijing, China, 2007.

[28] J. B. Li, Singular Nonlinear Traveling Wave Equations: Bifurcations and Exact Solutions, Science Press, Beijing, China, 2013.

[29] L. N. Zhang and J. B. Li, "Dynamical behavior of loop solutions for the $K(2,2)$ equation," Physics Letters A, vol. 375, no. 33, pp. 2965-2968, 2011.

[30] Z.-R. Liu and Y. Long, "Compacton-like wave and kinklike wave of GCH equation," Nonlinear Analysis: Real World Applications, vol. 8, no. 1, pp. 136-155, 2007.

[31] W. Yan and Z. Liu, "Some new nonlinear wave solutions and their convergence for the (2+1)-dimensional Broer-KauKupershmidt equation," Mathematical Methods in the Applied Sciences, vol. 38, no. 7, pp. 1303-1329, 2014.

[32] S. Y. Li and Z. R. Liu, "Kink-like wave and compacton-like wave solutions for generalized KdV equation," Nonlinear Dynamics, vol. 79, no. 2, pp. 903-918, 2014.

[33] B. He, "Bifurcations and exact bounded travelling wave solutions for a partial differential equation," Nonlinear Analysis: Real World Applications, vol. 11, no. 1, pp. 364-371, 2010.

[34] Q. Meng and B. He, "Notes on 'Solitary wave solutions of the generalized two component Hunter-Saxton system,' Nonlinear Analysis: Theory, Methods \& Applications, vol. 103, pp. 33-38, 2014.

[35] P. F. Byrd and M. D. Friedman, Handbook of Elliptic Integrals for Engineers and Scientists, Springer, Berlin, Germany, 1971. 


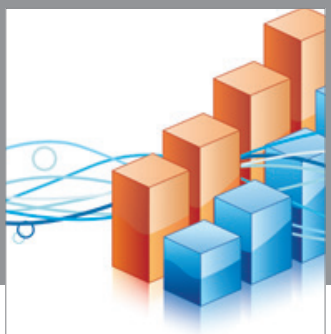

Advances in

Operations Research

vatem alat4

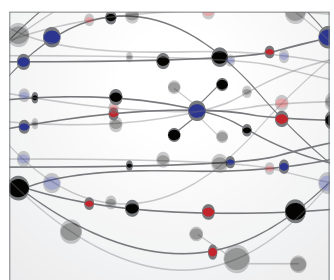

\section{The Scientific} World Journal
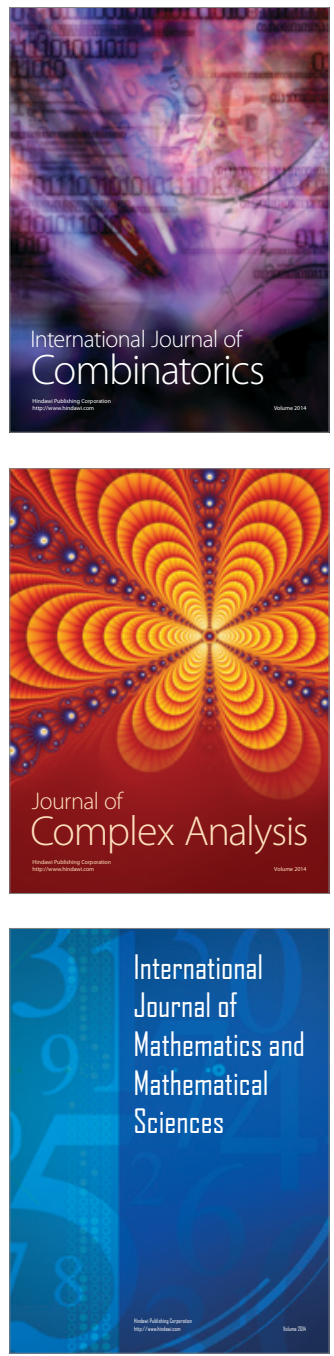
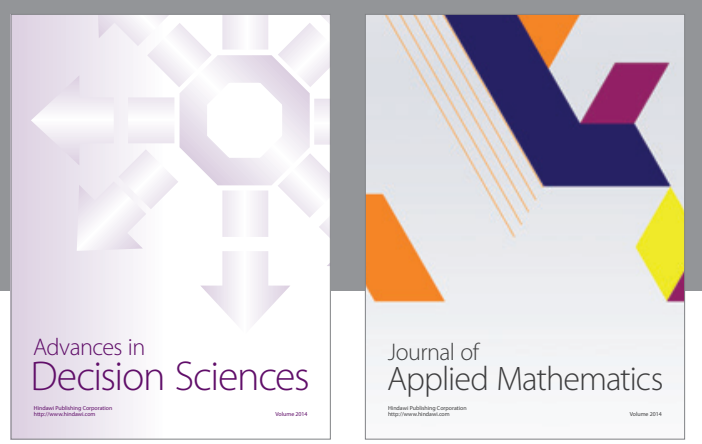

Algebra

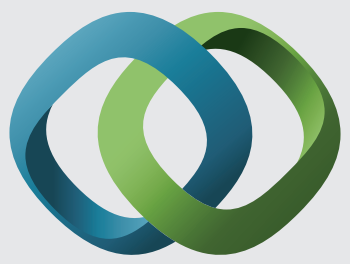

\section{Hindawi}

Submit your manuscripts at

http://www.hindawi.com
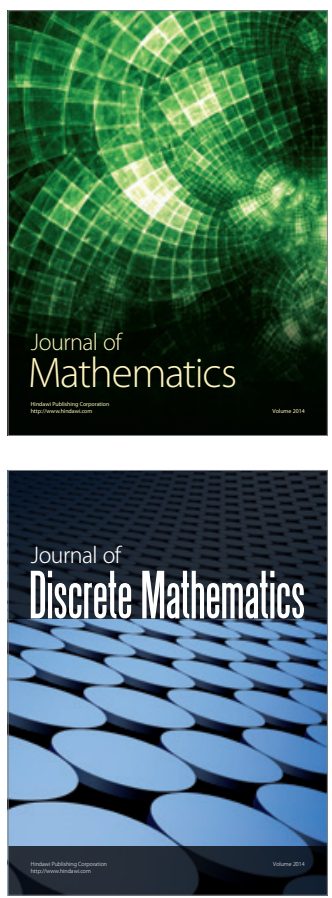

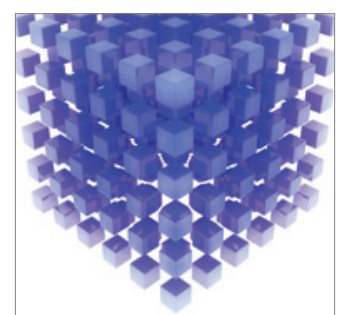

Mathematical Problems in Engineering
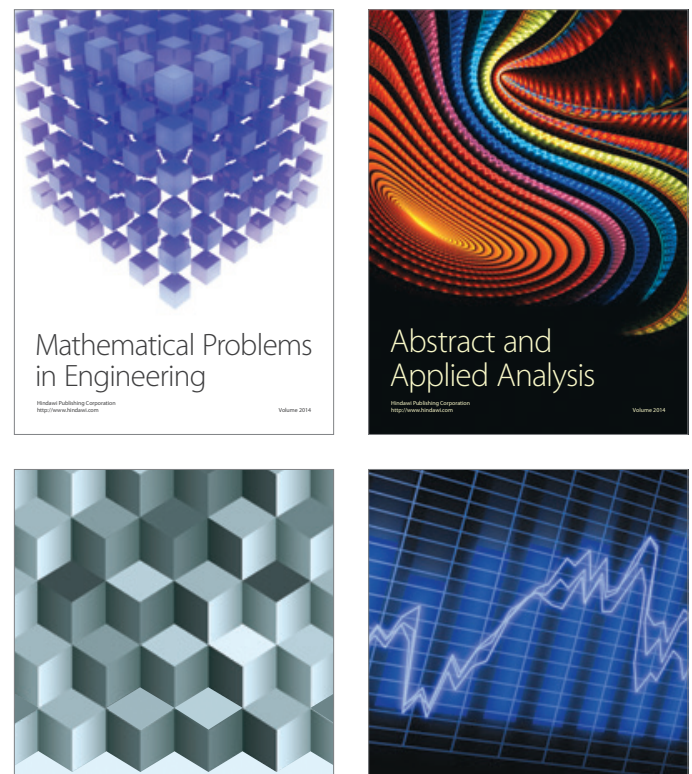

Journal of

Function Spaces

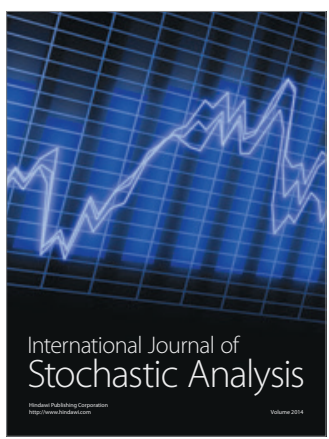

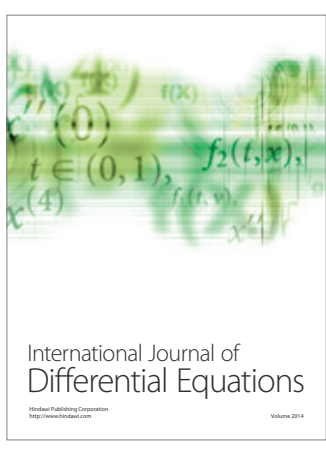
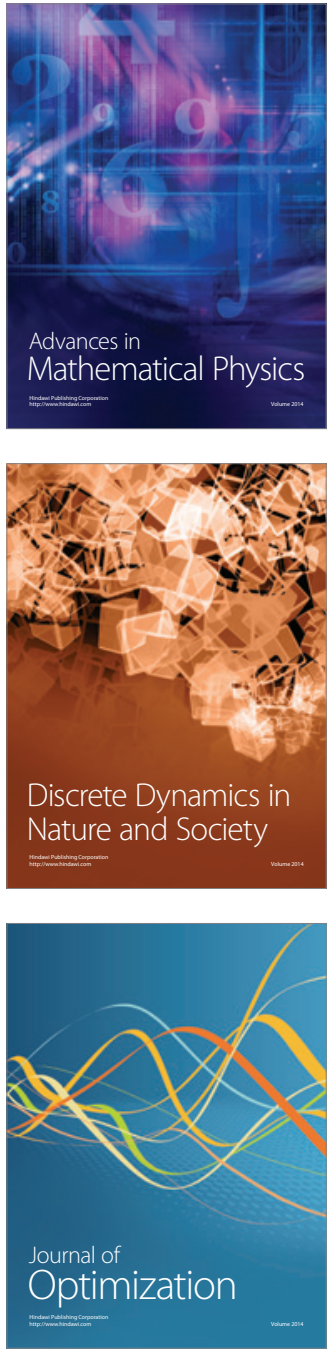